MATHEMATICS OF COMPUTATION

Volume 81, Number 278, April 2012, Pages 957-990

S $0025-5718(2011) 02531-7$

Article electronically published on August 15, 2011

\title{
SERIES EXPANSIONS OF SYMMETRIC ELLIPTIC INTEGRALS
}

\author{
TOSHIO FUKUSHIMA
}

\begin{abstract}
Based on general discussion of series expansions of Carlson's symmetric elliptic integrals, we developed fifteen kinds of them including eleven new ones by utilizing the symmetric nature of the integrals. Thanks to the special addition formulas of the integrals, we also obtained their complementary series expansions. By considering the balance between the speed of convergence and the amount of computational labor, we chose four of them as the best series expansions. Practical evaluation of the integrals is conducted by the most suitable one among these four series expansions. Its selection rule was analytically specified in terms of the numerical values of given parameters. As a by-product, we obtained an efficient asymptotic expansion of the integrals around their logarithmic singularities. Numerical experiments confirmed the effectiveness of these new series expansions.
\end{abstract}

\section{INTRODUCTION}

1.1. Symmetric elliptic integrals. Carlson reconstructed the theory of elliptic integrals by starting from two symmetric elliptic integrals [7, 8, 39, 9, 11, 12.

$$
\begin{gathered}
R_{F}(x, y, z) \equiv \frac{1}{2} \int_{0}^{\infty} \frac{d t}{\sqrt{(t+x)(t+y)(t+z)}}, \\
R_{J}(x, y, z, p) \equiv \frac{3}{2} \int_{0}^{\infty} \frac{d t}{(t+p) \sqrt{(t+x)(t+y)(t+z)}},
\end{gathered}
$$

where $x, y, z$, and $p$ are nonnegative parameters and at least one of $x, y$, and $z$ is positive. He prepared a concise and comprehensive summary of the symmetric and classic elliptic integrals in Chapter 19 of [35]; visit the website: http:// dlmf.nist.gov/19.

Besides $R_{F}(x, y, z)$ and $R_{J}(x, y, z, p)$, useful are two degenerate forms of them:

$$
\begin{aligned}
R_{D}(x, y, z) & \equiv R_{J}(x, y, z, z), \\
R_{C}(x, y) & \equiv R_{F}(x, y, y),
\end{aligned}
$$

where the latter is expressed by elementary functions. Refer to the discussion in $\S 19.16$ of 35 .

Received by the editor November 4, 2010 and, in revised form, January 30, 2011.

2010 Mathematics Subject Classification. Primary 33E05.

Key words and phrases. Elliptic integral.

(C)2011 American Mathematical Society Reverts to public domain 28 years from publication 
1.2. Relation with classic elliptic integrals. All elliptic integrals are finally expressed as a linear combination of $R_{F}(x, y, z)$ and $R_{J}(x, y, z, p)$. Indeed, the classic incomplete elliptic integrals are written in terms of them as

$$
\begin{gathered}
F(\varphi \mid m)=s R_{F}\left(c^{2}, d^{2}, 1\right), \\
E(\varphi \mid m)=s R_{F}\left(c^{2}, d^{2}, 1\right)-\left(\frac{m s^{3}}{3}\right) R_{D}\left(c^{2}, d^{2}, 1\right), \\
\Pi(\varphi, n \mid m)=s R_{F}\left(c^{2}, d^{2}, 1\right)+\left(\frac{n s^{3}}{3}\right) R_{J}\left(c^{2}, d^{2}, 1, f^{2}\right),
\end{gathered}
$$

where $\varphi$ is the amplitude, $m$ is the parameter, $n$ is the characteristic,

$$
m_{c} \equiv 1-m, \quad n_{c} \equiv 1-n,
$$

are the complementary parameter and characteristic, respectively, and

$$
s \equiv \sin \varphi, c \equiv \cos \varphi, d \equiv \sqrt{m_{c}+m c^{2}}, f \equiv \sqrt{n_{c}+n c^{2}} .
$$

As a corollary, the classic complete elliptic integrals are expressed as

$$
\begin{gathered}
K(m) \equiv F\left(\frac{\pi}{2} \mid m\right)=R_{F}\left(0, m_{c}, 1\right) \\
E(m) \equiv E\left(\frac{\pi}{2} \mid m\right)=R_{F}\left(0, m_{c}, 1\right)-\left(\frac{m}{3}\right) R_{D}\left(0, m_{c}, 1\right), \\
\Pi(n \mid m) \equiv \Pi\left(\frac{\pi}{2}, n \mid m\right)=R_{F}\left(0, m_{c}, 1\right)+\left(\frac{n}{3}\right) R_{J}\left(0, m_{c}, 1, n_{c}\right) .
\end{gathered}
$$

Refer to $\S 19.25$ of $[35]$.

1.3. Existing researches on series expansions of elliptic integrals. The series expansions of the classic elliptic integrals have been discussed mainly in order to seek a convenient way to evaluate them by means of the elementary functions such as the logarithm or inverse trigonometric functions [18, 21, 27, 19, 32, 38, 33, 2, 34. Nevertheless, these methods are known to be slower than: the standard methods utilizing Landen and/or Gauss transformations [3, 4, 5, 6, 26, 36. For example, our study [23] showed that the series expansion method for computing $F(\varphi \mid m)$ is significantly slower than: (1) Carlson's duplication method [12, 37, (2) Bulirsch's el1 based on the descending Landen transformation [3], and (3) our half argument transformation method 23 . On the other hand, recent research on the series expansions of the symmetric and classic elliptic integrals is instead emphasized to study the behavior of the integrals near their logarithmic singularities at $x=0$ and/or $y=0$ [13, 14, 30, 31, 20, 29, 28.

1.4. Contents of this article. During the course of our investigation on the fast procedures to compute $F(\varphi \mid m)$ in [23], we noticed that the methods based on the series expansions we chose are the slowest but the most precise. Also, it runs fairly fast when $\varphi$ is small, but it runs very slowly otherwise. We regard that this defect is due to the slowness of the convergence of the series expansions adopted. In order to resolve this issue of slow convergence, we will develop several new series expansions by using the symmetric nature of Carlson's basic integrals in this article.

First, we provide 15 series expansions by a new approach in $\$ 2$ Four of them are essentially the same as given in the literature and the other eleven are new. Next, in \$3, we: (1) discuss the effectiveness of these series expansions and their fifteen complements obtained from the introduction of special addition formulas, (2) 
choose four appropriate expansions among the 30 that were obtained and specify analytically the rule to select the best series expansion from the four candidates, (3) show an effective approach to compute the complete integrals by way of the incomplete ones, and (4) present algorithms to evaluate the complete and incomplete integrals, respectively. Finally, we illustrate some results of numerical experiments to confirm the effectiveness of the developed series expansions in $\$ 4$.

Besides, in Appendix A we describe some basic properties of the symmetric elementary integrals used in developing the series expansions. Also, in Appendix B, we derive the special addition formulas of the complete and incomplete symmetric elliptic integrals. Further, in Appendix C] we summarize the existing and new asymptotic series expansions of $F(\varphi \mid m)$.

\section{Development of SERIES EXPANSIONS}

In this section, we first describe a general approach to obtain a series expansion of elliptic and hyper-elliptic integrals in $\$ 2.1$. Second, we discuss the rate of convergence of the expansions in 2.2 . Third, we apply this approach to the symmetric elliptic integrals in 22.3 Finally, we develop four forms of their expansions and obtain fifteen series expansions: two in $\$ 2.4$, three in $\$ 2.5$, three in $\$ 2.6$, one in $\$ 2.7$, and six in $\$ 2.8$. Four of the last six series expansions are the same as the existing ones. The other eight are new.

2.1. Binomial expansion. Consider a real-valued integral containing the reciprocal square root of a polynomial as

$$
J \equiv \int_{t_{0}}^{t_{1}} \frac{R(t) d t}{Q(t) \sqrt{P(t)}}
$$

where $P(t), Q(t)$, and $R(t)$ are arbitrary polynomials. Depending on the order of $P(t)$, the integral is termed as elementary if the order is less than 3 , elliptic if it is 3 or 4 , and hyper-elliptic otherwise.

The base formula we use is the binomial expansion of the reciprocal square root,

$$
\frac{1}{\sqrt{1+x}}=\sum_{j=0}^{\infty}\left(\begin{array}{c}
-1 / 2 \\
j
\end{array}\right) x^{j} .
$$

Here the coefficients are directly expressed as

$$
\left(\begin{array}{c}
-1 / 2 \\
j
\end{array}\right)=\frac{(-1)^{j}(2 j-1) ! !}{(2 j) ! !}=\frac{(-1)^{j}(2 j-1)(2 j-3) \cdots 3 \cdot 1}{(2 j)(2 j-2) \cdots 4 \cdot 2}, \quad(j=1,2, \cdots)
$$

and

$$
\left(\begin{array}{c}
-1 / 2 \\
0
\end{array}\right)=1
$$

Let us split $P(t)$ as a sum of its approximate and the remainder as

$$
P(t)=P_{0}(t)+\Delta P(t) .
$$

Then, by conducting the binomial expansion with respect to their ratio, $\Delta P(t) / P_{0}(t)$, and exchanging the summation and the integration by assuming the convergence of the expansion, we obtain a series expansion of $J$ as

$$
J=\sum_{j=0}^{\infty} J_{j}
$$


where

$$
J_{j} \equiv\left(\begin{array}{c}
-1 / 2 \\
j
\end{array}\right) \int_{t_{0}}^{t_{1}}\left(\frac{\Delta P(t)}{P_{0}(t)}\right)^{j} \frac{R(t) d t}{Q(t) \sqrt{P_{0}(t)}} .
$$

The integral composing $J_{j}$ is expressed by elementary functions if: (1) $P_{0}(t)$ is at most quadratic, or (2) the number of simple or odd multiple roots of $P_{0}(t)$ is at most two such that the square root is rewritten as

$$
\sqrt{P_{0}(t)}=Q_{0}(t) \sqrt{P_{1}(t)}
$$

where $Q_{0}(t)$ is another polynomial and $P_{1}(t)$ is a polynomial of the order two or less.

2.2. Rate of convergence. Even if a series expansion is convergent, it does not automatically mean that the expansion is useful. The question is the rate of convergence. In case of the expansions discussed in the previous subsection, it depends on the manner of splitting of the given polynomial, $P(t)$, into the main term, $P_{0}(t)$, and the rest, $\Delta P(t)$. In fact, the expansion factor defined as the ratio of adjacent two terms in the series expansion is roughly estimated as

$$
e \equiv \frac{J_{j}}{J_{j-1}} \approx \max _{t_{0}<t<t_{1}}\left|\frac{\Delta P(t)}{P_{0}(t)}\right| .
$$

When the ratio $\left|\Delta P(t) / P_{0}(t)\right|$ can be large in the integral domain considered, it would result in a slow convergence or a divergence. For example, if $P_{0}(t)$ has a root within the integral domain, it generally leads to a divergence unless $R(t)$ also shares the same root, and therefore no pole remains.

The problem of convergence is only weakly related with the additional polynomials in the integrand, $Q(t)$ and/or $R(t)$. The exceptions are: (1) when the root(s) of $Q(t)$ introduces another singularity, and (2) when the $\operatorname{root}(\mathrm{s})$ of $R(t)$ removes the singularity caused by $P_{0}(t)$. Thus the speed of convergence is mostly insensitive with the forms of the additional polynomials. In this sense, the essential question lies only in the treatment of $R_{F}(x, y, z)$ in case of the symmetric elliptic integrals.

2.3. Application to symmetric elliptic integrals. In symmetric elliptic integrals, $P(t)$ is a cubic polynomial with three nonpositive roots as

$$
P(t)=(t+x)(t+y)(t+z)=t^{3}+E_{1} t^{2}+E_{2} t+E_{3},
$$

where

$$
E_{1} \equiv x+y+z, \quad E_{2} \equiv x y+y z+z x, \quad E_{3} \equiv x y z,
$$

are elementary symmetric functions of three parameters, $x, y$, and $z$. Refer to $\S 19.19$ of 35 .

Hereafter, we assume that $x$ and $y$ are nonnegative, $z$ is positive, and they are ordered as

$$
0 \leq x \leq y \leq z .
$$

This condition is always satisfied by, if necessary, an appropriate permutation of the parameters and their renaming. Therefore, the assumption will not lose the generality in the discussions below.

The integral domain is $(0, \infty)$ in symmetric elliptic integrals. Then we limit ourselves to the case where $P_{0}(t)$ is cubic and $\Delta P(t)$ is at most quadratic. If not, the cubic term in $\Delta P(t)$ would cause divergence of the expansions. At any rate, 
the approximation is limited to two types: (1) $P_{0}(t)=(t+u)(t+v)^{2}$ where $u \neq v$, or $(2) P_{0}(t)=(t+u)^{3}$.

Even after this restriction, there remain many possibilities to split $P(t)$. Since $P(t)$ includes three parameters, there exists no way of splitting containing only two or less parameters. If it has three parameters, there may or may not be real solution(s) to satisfy the splitting condition. On the other hand, if it contains four or more parameters, they are generally indeterminate. More specifically speaking, some of them would be free parameters. Since a general discussion including such indeterminate cases would be too complicated, we will examine below only the determinate cases.

2.4. Constant remainder of first type. Let us begin with the case when $\Delta P(t)$ is a constant. The second type splitting cannot satisfy the splitting condition since it contains only two parameters. Then we look for a solution of the first type as

$$
P_{0}(t)=(t+u)(t+v)^{2}, \Delta P(t)=a,
$$

where $u, v$, and $a$ are new parameters to be determined from the original parameters $x, y$, and $z$. If we find a solution satisfying the splitting condition, equation (2.5), then $R_{F}(x, y, z), R_{D}(x, y, z)$, and $R_{J}(x, y, z, p)$ are expanded with respect to $a$ as

$$
\begin{gathered}
R_{F}(x, y, z)=\sum_{j=0}^{\infty}\left(\begin{array}{c}
-1 / 2 \\
j
\end{array}\right) a^{j} I_{j, 2 j+1}(u, v), \\
R_{D}(x, y, z)=3 \sum_{j=0}^{\infty}\left(\begin{array}{c}
-1 / 2 \\
j
\end{array}\right) a^{j} I_{j, 2 j+1,1}(u, v, z), \\
R_{J}(x, y, z, p)=3 \sum_{j=0}^{\infty}\left(\begin{array}{c}
-1 / 2 \\
j
\end{array}\right) a^{j} I_{j, 2 j+1,1}(u, v, p),
\end{gathered}
$$

where

$$
I_{j k \ell}(x, y, z) \equiv \frac{1}{2} \int_{0}^{\infty} \frac{d t}{(t+x)^{j}(t+y)^{k}(t+z)^{\ell} \sqrt{t+x}},
$$

is an auxiliary symmetric integral with three parameters and

$$
I_{j k}(x, y) \equiv I_{j k 0}(x, y,-)=\frac{1}{2} \int_{0}^{\infty} \frac{d t}{(t+x)^{j}(t+y)^{k} \sqrt{t+x}},
$$

is its degenerate form with two parameters. These auxiliary integrals are expressed in terms of elementary functions as given in Appendix A The expansion factor is roughly approximated as

$$
e \approx \frac{|a|}{u v^{2}}
$$

since the main part of the ratios $I_{j, k+1}(x, y) / I_{01}(x, y)$ and $I_{j, k+1,1}(x, y, z) / I_{011}(x, y, z)$ is roughly in proportion to $1 /\left(x^{j} y^{k}\right)$.

Let us return to finding solutions to satisfy the splitting condition, equation (2.5). The condition is rewritten into three equations with respect to the new parameters as

$$
u+2 v=E_{1}, \quad 2 u v+v^{2}=E_{2}, \quad u v^{2}+a=E_{3} .
$$

These reduce to a quadratic equation with respect to $v$ as

$$
3 v^{2}-2 E_{1} v+E_{2}=0
$$


Its quarter discriminant is nonnegative as

$$
D \equiv E_{1}^{2}-3 E_{2}=\frac{(x-y)^{2}}{2}+\frac{(y-z)^{2}}{2}+\frac{(z-x)^{2}}{2} \geq 0 .
$$

We may exclude the case $D=0$ since it means $x=y=z$, and therefore the evaluation of the symmetric elliptic integrals is as trivial as

$$
R_{F}(x, x, x)=\frac{1}{\sqrt{x}}, \quad R_{D}(x, x, x)=\frac{1}{x \sqrt{x}}, \quad R_{J}(x, x, x, p)=I_{11}(x, p),
$$

where $I_{11}(x, y)$ is an elementary function explicitly given by equation (A.11) in Appendix A

Thus we assume that $D>0$. Then the equations have two real solutions. One of them is

$$
u_{1}=\frac{E_{1}+2 \sqrt{D}}{3}, \quad v_{1}=\frac{E_{2}}{E_{1}+\sqrt{D}}, \quad a_{1}=\frac{C-2 D \sqrt{D}}{27},
$$

and the other one is

$$
u_{2}=\frac{4 E_{2}-E_{1}^{2}}{E_{1}+2 \sqrt{D}}, \quad v_{2}=\frac{E_{1}+\sqrt{D}}{3}, \quad a_{2}=\frac{C+2 D \sqrt{D}}{27},
$$

where

$$
C \equiv 2 E_{1}^{3}-9 E_{1} E_{2}+27 E_{3},
$$

is an auxiliary symmetric polynomial of the third order.

It is easy to show that

$$
u_{1}-v_{1}=-\left(u_{2}-v_{2}\right)=\sqrt{D}>0,
$$

and the variables except $u_{2}$ do not change the sign as

$$
\begin{aligned}
& 0<z \leq u_{1} \leq \frac{4 z}{3}, \quad 0 \leq v_{1} \leq z,-\left(\frac{4 z^{3}}{27}\right) \leq a_{1} \leq 0, \\
& -\left(\frac{z}{3}\right) \leq u_{2} \leq z, \quad 0<\frac{2 z}{3} \leq v_{2} \leq z, \quad 0 \leq a_{2} \leq \frac{4 z^{3}}{27} .
\end{aligned}
$$

Note that $v_{1}=0$ only when $x=y=0$. Also, $u_{2}$ becomes nonpositive when $\sqrt{x}+\sqrt{y} \leq \sqrt{z}$. In that case, the series expansion based on the second solution will be inadequate since $\sqrt{t+u_{2}}$ may become purely imaginary.

2.5. Linear remainder of first type: case A. Next, we assume that $\Delta P(t)$ is a linear function of $t$. The determinate splitting form of the first type becomes

$$
P_{0}(t)=(t+u)(t+v)^{2}, \Delta P(t)=a(t+w),
$$

where $w=u$ or $w=v$. First, we consider the case $w=u$. Then the resulting expansions of the integrals are written as

$$
\begin{gathered}
R_{F}(x, y, z)=\sum_{j=0}^{\infty}\left(\begin{array}{c}
-1 / 2 \\
j
\end{array}\right) a^{j} I_{0,2 j+1}(u, v), \\
R_{D}(x, y, z)=3 \sum_{j=0}^{\infty}\left(\begin{array}{c}
-1 / 2 \\
j
\end{array}\right) a^{j} I_{0,2 j+1,1}(u, v, z), \\
R_{J}(x, y, z, p)=3 \sum_{j=0}^{\infty}\left(\begin{array}{c}
-1 / 2 \\
j
\end{array}\right) a^{j} I_{0,2 j+1,1}(u, v, p) .
\end{gathered}
$$


The approximate expansion factor becomes

$$
e \approx \frac{|a|}{v^{2}}
$$

There are three solutions to satisfy the splitting condition:

$$
\begin{aligned}
& u_{3}=x, \quad v_{3}=\frac{y+z}{2}, \quad a_{3}=-\left(\frac{z-y}{2}\right)^{2}, \\
& u_{4}=y, \quad v_{4}=\frac{x+z}{2}, \quad a_{4}=-\left(\frac{z-x}{2}\right)^{2}, \\
& u_{5}=z, \quad v_{5}=\frac{x+y}{2}, \quad a_{5}=-\left(\frac{y-x}{2}\right)^{2} .
\end{aligned}
$$

Obviously, (1) $u_{3}, u_{4}$, and $v_{5}$ are nonnegative, (2) $v_{3}, v_{4}$, and $u_{5}$ are positive, and (3) $a_{3}, a_{4}$, and $a_{5}$ are nonpositive. There are the inequalities

$$
u_{3} \leq v_{3}, u_{5} \geq v_{5},
$$

meanwhile $u_{4}$ can be greater than, equal to, or smaller than $v_{4}$.

The auxiliary integrals used to evaluate $R_{D}$ by the third solution $\left(u_{5}, v_{5}, a_{5}\right)$ are simplified as

$$
I_{0,2 j+1,1}\left(u_{5}, v_{5}, z\right)=I_{1,2 j+1}\left(z, \frac{x+y}{2}\right),
$$

since $u_{5}=z$.

2.6. Linear remainder of first type: case B. The next case is when $w=v$. This time, the expansions of the integrals become

$$
\begin{gathered}
R_{F}(x, y, z)=\sum_{j=0}^{\infty}\left(\begin{array}{c}
-1 / 2 \\
j
\end{array}\right) a^{j} I_{j, j+1}(u, v), \\
R_{D}(x, y, z)=3 \sum_{j=0}^{\infty}\left(\begin{array}{c}
-1 / 2 \\
j
\end{array}\right) a^{j} I_{j, j+1,1}(u, v, z), \\
R_{J}(x, y, z, p)=3 \sum_{j=0}^{\infty}\left(\begin{array}{c}
-1 / 2 \\
j
\end{array}\right) a^{j} I_{j, j+1,1}(u, v, p) .
\end{gathered}
$$

The approximate expansion factor is expressed as

$$
e \approx \frac{|a|}{u v} \text {. }
$$

Again, there are three solutions to satisfy the splitting condition:

$$
\begin{aligned}
& u_{6}=y+z-x, \quad v_{6}=x, \quad a_{6}=(z-x)(y-x), \\
& u_{7}=z+x-y, \quad v_{7}=y, \quad a_{7}=(x-y)(z-y), \\
& u_{8}=x+y-z, \quad v_{8}=z, \quad a_{8}=(y-z)(x-z),
\end{aligned}
$$

Obviously, $u_{6}, u_{7}, v_{6}, v_{7}, v_{8}, a_{6}$, and $a_{8}$ are nonnegative while $a_{7}$ is nonpositive. When $x+y<z, u_{8}$ is negative, and therefore this solution becomes inappropriate since $\sqrt{t+u_{8}}$ may be imaginary. 
Again, the auxiliary integrals used to evaluate $R_{D}$ by the third solution $\left(u_{8}, v_{8}, a_{8}\right)$ are simplified as

$$
I_{j, j+1,1}\left(u_{8}, v_{8}, z\right)=I_{j, j+2}\left(u_{8}, z\right),
$$

since $v_{8}=z$.

2.7. Linear remainder of second type. Let us move to the case of the linear remainder with the second type solution as

$$
P_{0}(t)=(t+u)^{3}, \quad \Delta P(t)=a(t+u)+b .
$$

This time, the integrals are expanded as

$$
\begin{gathered}
R_{F}(x, y, z)=\sum_{j=0}^{\infty} A_{j}(a, b) I_{j+1}(u), \\
R_{D}(x, y, z)=3 \sum_{j=0}^{\infty} A_{j}(a, b) I_{j+1,1}(u, z), \\
R_{J}(x, y, z, p)=3 \sum_{j=0}^{\infty} A_{j}(a, b) I_{j+1,1}(u, p),
\end{gathered}
$$

where

$$
I_{j}(x) \equiv I_{j 00}(x,-,-)=I_{j 0}(x,-)=\frac{1}{2} \int_{0}^{\infty} \frac{d t}{(t+x)^{j} \sqrt{t+x}},
$$

is a degenerate form of $I_{j k \ell}(x, y, z)$ with one parameter, and

$$
A_{j}(a, b) \equiv \sum_{k=\lceil j / 3\rceil}^{\lfloor j / 2\rfloor}\left(\begin{array}{c}
-1 / 2 \\
k
\end{array}\right)\left(\begin{array}{c}
k \\
3 k-j
\end{array}\right) a^{3 k-j} b^{j-2 k},
$$

is a bivariate polynomial. Some low order expressions of $A_{j}(a, b)$ are

$$
\begin{array}{r}
A_{0}=1, \quad A_{1}=0, A_{2}=\frac{-a}{2}, A_{3}=\frac{-b}{2}, A_{4}=\frac{3 a^{2}}{8}, A_{5}=\frac{3 a b}{4}, \\
A_{6}=\frac{6 b^{2}-5 a^{3}}{16}, A_{7}=\frac{-15 a^{2} b}{16}, A_{8}=\frac{35 a^{4}-120 a b^{2}}{128}, \cdots
\end{array}
$$

A very rough estimate of the expansion factor is given as

$$
e \sim \frac{|a| u+|b|}{u^{3}} .
$$

Solving similar equations with respect to the new parameters, $u, a$, and $b$, we obtain a unique solution,

$$
u_{9}=\frac{E_{1}}{3}, \quad a_{9}=\frac{-D}{3}, \quad b_{9}=\frac{C}{27},
$$

where $D$ and $C$ are already given in equations (2.22) and (2.26), respectively.

We note that $u_{9}$ is positive and $a_{9}$ is nonpositive while $b_{9}$ changes the sign as

$$
0<\frac{z}{3} \leq u_{9} \leq z,-\left(\frac{z^{2}}{3}\right) \leq a_{9} \leq 0, \quad\left|b_{9}\right| \leq \frac{2 z^{3}}{27} .
$$

In fact, $b_{9}<0$ when $2 y<x+z$ and vice versa. 
2.8. Quadratic remainder of first type. Finally, we assume that $\Delta P(t)$ is quadratic. We consider only the determinate case. It is of the first type as

$$
P_{0}(t)=(t+u)(t+v)^{2}, \quad \Delta P(t)=a(t+u)(t+v) .
$$

Then the integrals are expanded as:

$$
\begin{gathered}
R_{F}(x, y, z)=\sum_{j=0}^{\infty}\left(\begin{array}{c}
-1 / 2 \\
j
\end{array}\right) a^{j} I_{0, j+1}(u, v), \\
R_{D}(x, y, z)=\sum_{j=0}^{\infty}\left(\begin{array}{c}
-1 / 2 \\
j
\end{array}\right) a^{j} I_{0, j+1,1}(u, v, z), \\
R_{J}(x, y, z, p)=\sum_{j=0}^{\infty}\left(\begin{array}{c}
-1 / 2 \\
j
\end{array}\right) a^{j} I_{0, j+1,1}(u, v, p) .
\end{gathered}
$$

The expansion factor is roughly evaluated as

$$
e \approx \frac{|a|}{v} \text {. }
$$

The following six solutions satisfy the splitting condition:

$$
\begin{array}{ll}
u_{10}=x, & v_{10}=y, \quad a_{10}=z-y, \\
u_{11}=x, & v_{11}=z, \quad a_{11}=y-z, \\
u_{12}=y, & v_{12}=x, \quad a_{12}=z-x, \\
u_{13}=y, & v_{13}=z, \quad a_{13}=x-z, \\
u_{14}=z, & v_{14}=x, \quad a_{14}=y-x, \\
u_{15}=z, & v_{15}=y, \quad a_{15}=x-y .
\end{array}
$$

Apparently, the difference between the choice of $u_{10}, v_{10}$, and $a_{10}$ and that of $u_{11}$, $v_{11}$, and $a_{11}$ is small. The same is said for that of the 12 th and 13th solutions and that of the 14th and 15th solutions. In some cases, the auxiliary integrals used to evaluate $R_{D}$ are simplified as

$$
\begin{array}{ll}
I_{0, j+1,1}\left(u_{11}, v_{11}, z\right)=I_{0, j+2}(x, z), & I_{0, j+1,1}\left(u_{13}, v_{13}, z\right)=I_{0, j+2}(y, z), \\
I_{0, j+1,1}\left(u_{14}, v_{14}, z\right)=I_{1, j+1}(z, x), & I_{0, j+1,1}\left(u_{15}, v_{15}, z\right)=I_{1, j+1}(z, y),
\end{array}
$$

since $u_{11}=v_{14}=x, u_{13}=v_{15}=y$, and $v_{11}=v_{13}=u_{14}=u_{15}=z$.

Some of these series expansions are essentially the same as the classic ones given in the literature [2. In fact, (1) the series expansions of $R_{F}(x, y, z), R_{D}(x, y, z)$, and $R_{J}(x, y, z, p)$ using $\left(u_{11}, v_{11}, a_{11}\right)$ reduce to those of $F(\varphi \mid m), E(\varphi \mid m), \Pi(n \mid m)$, and $\Pi(\varphi, n \mid m)$ with respect to $m \sin ^{2} \varphi$ given as the formulas 902.00,903.00, 906.00, and 906.01 of [2], (2) those of $R_{F}(x, y, z)$ and $R_{D}(x, y, z)$ using $\left(u_{14}, v_{14}, a_{14}\right)$ reduce to those of $F(\varphi \mid m)$ and $E(\varphi \mid m)$ with respect to $m_{c} \tan ^{2} \varphi$ given as the formulas 902.01 and 903.01 of [2], and (3) those using $\left(u_{12}, v_{12}, a_{12}\right)$ and $\left(u_{13}, v_{13}, a_{13}\right)$ reduce to the Maclaurin series expansions of the classic integrals in terms of $\tan ^{2} \varphi$ and $\sin ^{2} \varphi$, respectively. Both of the last two group of series expansions further reduce to those of the classic integrals in terms of $\varphi$ by using the Maclaurin series expansions of $\tan \varphi$ or $\sin \varphi$ with respect to $\varphi$. 


\section{Selection of Best formulas}

Let us discuss the effectiveness of the series expansions we obtained in the previous section. First, in 3.1 we compare the magnitude of approximate expansion factors by regarding them as an index of the rate of convergence. Second, in $₫ 3.2$, we examine the amount of computational labor required. Third, in \$3.3. we introduce the special addition formulas of the integrals as a remedy to overcome the problem of slow convergence when the integrals are close to being complete. Fourth, in $\$ 3.4$, we introduce the complementary parameters and expansion factors to be used with the special addition formulas. Since each series expansion we obtained accompanies its complement, we finally have 30 different series expansions. Fifth, in 93.5 , we find the best combination among these series expansions. The usage of the special addition formulas assumes the availability of another procedure to evaluate complete integrals. As its realization, we develop a formulation to compute the complete integrals by way of the associated incomplete ones in $\$ 3.6$. Finally, in 3.7 . we present algorithms to calculate the three symmetric elliptic integrals simultaneously both for the complete and the incomplete cases.

3.1. Magnitude of expansion factor. In order to discuss the effectiveness of the series expansions we presented, we first compare the magnitude of their approximate expansion factors. The smaller it is, the faster the corresponding series expansion would converge. Thus we shall search for the minimum expansion factor. Of course, these factors are of approximate nature. Therefore we note that the result will be also of approximate nature.

Recall that the expansion factor, $e$, defined in $\$ 2.2$ is generally a function of the index $j$ as well as the integral arguments $x, y, z$, and/or $p$, but not the integral variable, $t$. In fact, equation (2.9) simply approximates it by the maximum value of the absolute ratio of two polynomials, $\Delta P(t) / P_{0}(t)$, in the integrand. Then it is independent on $t$ as well as on the index $j$.

At any rate, the expansion factors we obtained in the previous section are summarized as

$$
\begin{gathered}
e_{1}=\frac{C-2 D \sqrt{D}}{27 E_{3}-(C-2 D \sqrt{D})}, \quad e_{2}=\frac{C+2 D \sqrt{D}}{27 E_{3}-(C+2 D \sqrt{D})}, \\
e_{3}=\left(\frac{z-y}{z+y}\right)^{2}, \quad e_{4}=\left(\frac{z-x}{z+x}\right)^{2}, e_{5}=\left(\frac{y-x}{y+x}\right)^{2}, \\
e_{6}=\frac{(z-x)(y-x)}{x(y+z-x)}, \quad e_{7}=\frac{(z-y)(y-x)}{y(z+x-y)}, \quad e_{8}=\frac{(z-y)(z-x)}{z|x+y-z|}, \\
e_{10}=\frac{z-y}{y}, \quad e_{11}=\frac{3 E_{1} D+|C|}{E_{1}^{3}}, \quad e_{12}=\frac{z-x}{x} \\
e_{13}=\frac{z-x}{z}, \quad e_{14}=\frac{y-x}{x}, \quad e_{15}=\frac{y-x}{y} .
\end{gathered}
$$

All the factors are functions of $x / z$ and/or $y / z$ only. Especially $e_{4}, e_{12}$, and $e_{13}$ are independent on $y$. This means that the convergence of the corresponding series expansions of $F(\varphi \mid m), E(\varphi \mid m)$, and $\Pi(\varphi, n \mid m)$ is governed by $\varphi$ only. 


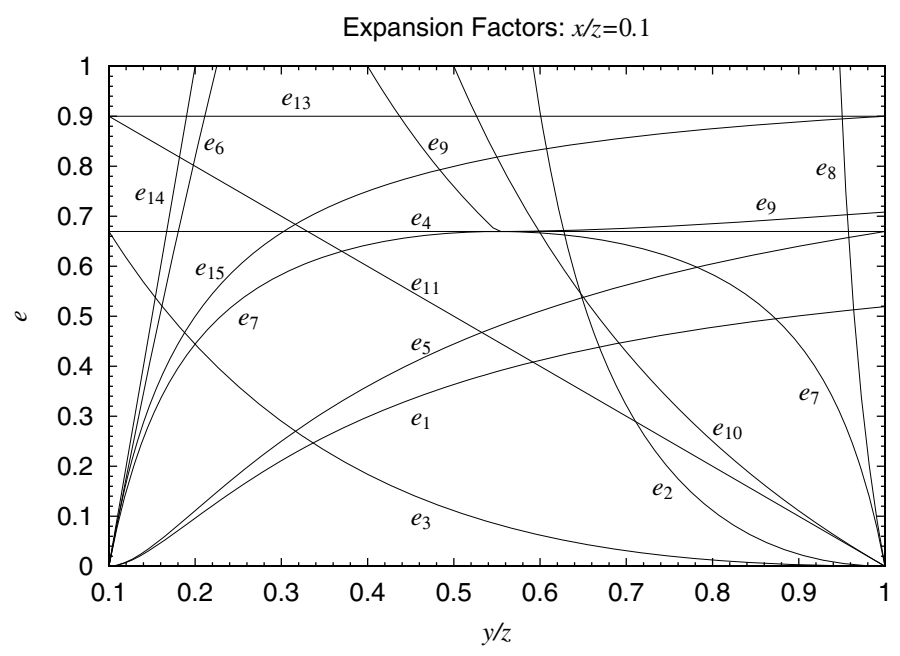

FiguRE 1. Factors of various series expansions. Shown are the expansion factors $e_{1}$ through $e_{15}$ as functions of $y / z$ while $x / z$ is fixed as 0.1. The curve of $e_{9}$ kinks at $(y / z, e) \sim(0.55,0.67)$. Meanwhile that of $e_{12}$ is outside of the drawn region.

The above expressions lead to the inequalities among them:

$$
\begin{gathered}
e_{1} \leq e_{5} \leq e_{15} \leq e_{6} \leq e_{14}, \quad e_{3} \leq e_{2}, \quad e_{3} \leq e_{4} \leq e_{13} \leq e_{12}, \\
e_{3} \leq e_{9}, \quad e_{3} \leq e_{11} \leq e_{10} \leq e_{8}, \quad e_{7} \leq e_{6}, \quad e_{7} \leq e_{8}, \quad e_{7} \leq e_{4},
\end{gathered}
$$

where we regard $e_{2}$ as its positive value. See Figure 1 for an illustration of these inequalities. It shows the expansion factors $e_{1}$ through $e_{15}$ as functions of $y / z$ when $x / z=0.1$. The curve of $e_{12}$ is scaled out of the figure.

The above inequalities tell us that the minimum expansion factor is the minimum of $e_{1}$ and $e_{3}$ and the second minimum is realized by the minimum of $e_{3}$ and $e_{5}$. The difference between these two minima is not so large. See Figure 2, Both minima are unimodal with respect to $y / z$ when $x / z$ is fixed and are monotonically decreasing with respect to $x / z$ when $y / z$ is fixed. Their maximum values are around unity and are achieved when $x / z \sim y / z \sim 0$. This indicates that these series expansions face with the problem of slow convergence near the logarithmic singularity, $x / z=y / z=0$.

3.2. Complexity of series computation. All the series expansions we developed are composed of single summations. Then the complexity of their computation depends on two factors: (1) the number of terms needed to assure a certain level of precision, and (2) the complexity of computation of each term. The former factor is controlled by the magnitude of the expansion factors, which we already discussed in the previous subsection. Thus we concentrate ourselves to the latter factor here. Below, we examine its components one by one.

First, the binomial coefficients consist of a one-dimensional array. The number of its components required is at most 30 or less. Also, they are effectively computed 


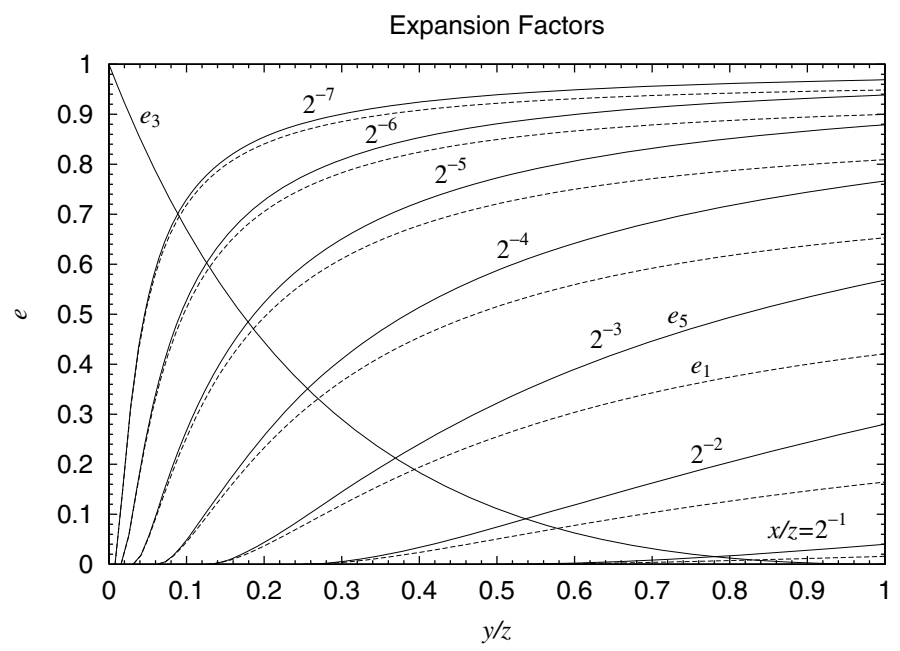

Figure 2. Factors of efficient series expansions. Shown are the expansion factors $e_{1}, e_{3}$, and $e_{5}$ as functions of $y / z$ while $x / z$ is fixed. The curves of $e_{1}$ and $e_{5}$ are shown for various values of $x / z$ meanwhile that of $e_{3}$ is independent on $x / z$. The curves of $e_{3}$ and $e_{5}$ are drawn as solid lines while those of $e_{1}$ are broken lines. Note that meaningful is the region $x \leq y$.

by a recurrence formula

$$
\left(\begin{array}{c}
-1 / 2 \\
j
\end{array}\right)=\frac{1-2 j}{2 j}\left(\begin{array}{c}
-1 / 2 \\
j-1
\end{array}\right),
$$

with the initial condition

$$
\left(\begin{array}{c}
-1 / 2 \\
0
\end{array}\right)=1
$$

Then it is easy to compute them beforehand and store their numerical values as predefined constants in computational codes. Therefore this component has no practical contribution to the complexity.

Second, the evaluation of the new parameters requires nonnegligible amount of computational time only in cases of $\left(u_{1}, v_{1}, a_{1}\right)$ and $\left(u_{2}, v_{2}, a_{2}\right)$. There one call of the square root is needed in computing $\sqrt{D}$. This computational overhead is small.

Third, we need to compute a bivariate polynomial $A_{j}(a, b)$ in the case of $\left(u_{9}, a_{9}, b_{9}\right)$. Its number of terms increases quadratically with respect to its degree $j$. Then this causes significant increase in its computational labor.

Finally, the main computational work is the preparation of auxiliary integrals, $I_{j}, I_{j k}$, and/or $I_{j k \ell}$. As will be explained later in Appendix A their computation mainly consists of two parts: the computation of $R_{C}(x, y)$ and the application of recurrence formulas. The first part requires an evaluation of one transcendental function: the logarithm or the inverse tangent. Nevertheless, it is common to all the cases except the computation of $R_{F}$ in case of $\left(u_{9}, a_{9}, b_{9}\right)$. Then it makes no practical difference. 
The second part is important. In the cases of $\left(u_{1}, v_{1}, a_{1}\right)$ and $\left(u_{2}, v_{2}, a_{2}\right)$, we need to compute $I_{j, 2 j+1}$ and $I_{j, 2 j+1,1}$. Also, we have to calculate $I_{j, j+1}$ and $I_{j, j+1,1}$ in the case of $\left(u_{6}, v_{6}, a_{6}\right)$ through $\left(u_{8}, v_{8}, a_{8}\right)$. These computational labors increase quadratically with respect to the index $j$. This requires a significant number of arithmetic operations when the maximum index to be considered amounts to 13-57 as will be estimated later. Meanwhile, the other cases demand: (1) the pair of $I_{0,2 j+1}$ and $I_{0,2 j+1,1}$ in the case of $\left(u_{3}, v_{3}, a_{3}\right)$ through $\left(u_{5}, v_{5}, a_{5}\right),(2)$ the pair of $I_{j+1}$ and $I_{j+1,1}$ in the case of $\left(u_{9}, a_{9}, b_{9}\right)$, or (3) the pair of $I_{0, j+1}$ and $I_{0, j+1,1}$ in the case of $\left(u_{10}, v_{10}, a_{10}\right)$ through $\left(u_{15}, v_{15}, a_{15}\right)$. The computational labor of these integrals increases only linearly with respect to $j$. Thus the best series expansions in terms of the computational labor are those using $\left(u_{3}, v_{3}, a_{3}\right)$ through $\left(u_{5}, v_{5}, a_{5}\right)$ or $\left(u_{10}, v_{10}, a_{10}\right)$ through $\left(u_{15}, v_{15}, a_{15}\right)$. Next comes that using $\left(u_{9}, a_{9}, b_{9}\right)$, then $\left(u_{6}, v_{6}, a_{6}\right)$ through $\left(u_{8}, v_{8}, a_{8}\right)$, and the most complicated are those using $\left(u_{1}, v_{1}, a_{1}\right)$ or $\left(u_{2}, v_{2}, a_{2}\right)$.

By combining the observations in this and the previous subsections, we conclude that the most efficient series expansions are those using $\left(u_{3}, v_{3}, a_{3}\right)$ or $\left(u_{5}, v_{5}, a_{5}\right)$. The choice between these two depends on the smallness of the expansion factors $e_{3}$ and $e_{5}$ for the given parameters $x, y$, and $z$.

3.3. Special addition formulas. As we see in the previous subsections, the series expansions we developed become inefficient when $x / z$ and $y / z$ are small. Of course, the expansion factors of some series expansions such as $e_{1}, e_{3}$, or $e_{5}$ remain to be less than unity in most cases. Then these series expansions generally converge in principle. Nevertheless, their convergence is so slow in some cases that we cannot regard them as practical procedures to calculate the integrals. However, there is a remedy [18, 21, 27, 19, 32, 38.

The special case when $x=0$ leads to the complete integrals. If their values are available by other approaches such as provided in the literatures $[3,15,16,17,22$, 24, 25. or as will be described later in $\$ 3.6$, there is another way to compute the incomplete integrals by the following formulas:

$$
\begin{gathered}
R_{F}(x, y, z)=R_{F}(0, y-x, z-x)-\sqrt{x} R_{F}\left(x_{c}, y_{c}, z_{c}\right), \\
R_{D}(x, y, z)=R_{D}(0, y-x, z-x)-x \sqrt{x} R_{D}\left(x_{c}, y_{c}, z_{c}\right)-\left(\frac{3}{z-x}\right) \sqrt{\frac{x}{y z}} \\
R_{J}(x, y, z, p)=R_{J}(0, y-x, z-x, p-x)-x \sqrt{x} R_{J}\left(x_{c}, y_{c}, z_{c}, p_{c}\right) \\
-3\left(\sqrt{\frac{x}{p-x}}\right) R_{C}\left(y z(p-x), p p_{c}\right),
\end{gathered}
$$

where

$$
x_{c} \equiv(y-x)(z-x), \quad y_{c} \equiv z(y-x), \quad z_{c} \equiv y(z-x), \quad p_{c} \equiv x(p-x)+x_{c},
$$

are the complementary parameters satisfying the similar order condition as the original one as

$$
0 \leq x_{c} \leq y_{c} \leq z_{c} .
$$

The above expressions are rewritings of the addition theorem of the symmetric elliptic integrals provided as the formulas 19.26.1, 19.26.7, and 19.26.9 of [35]. Refer to Appendix B.2 for the rewriting. Classically, these are called the special addition formulas. Refer to the formulas 116.01 through 116.03 of [2] and the formulas 19.11.7, 19.11.8, and 19.11 .10 of 35 . 
3.4. Complementary expansion factors. By means of the special addition formulas provided in the previous subsection, we may overcome the problem of small ratios of $x / z$ and $y / z$. The corresponding ratios in terms of complementary parameters become

$$
\frac{x_{c}}{z_{c}}=\frac{y-x}{y}, \frac{y_{c}}{z_{c}}=\frac{z(y-x)}{y(z-x)} .
$$

Even when $x / z$ and $y / z$ are small, these are not generally small, and therefore the series expansions may become efficient again. In order to examine this situation more clearly, let us denote the new parameters and expansion factors in terms of the complementary parameters by adding the suffix $c$.

Then the complementary expansion factors are summarized as

$$
\begin{gathered}
e_{1 c}=\frac{C_{c}-2 D_{c} \sqrt{D_{c}}}{27 E_{3 c}-\left(C_{c}-2 D_{c} \sqrt{D_{c}}\right)}, \quad e_{2 c}=\frac{C_{c}+2 D_{c} \sqrt{D_{c}}}{27 E_{3 c}-\left(C_{c}+2 D_{c} \sqrt{D_{c}}\right)}, \\
e_{3 c}=\left(\frac{(z-y) x}{2 y z-(y+z) x}\right)^{2}, \quad e_{4 c}=\left(\frac{x}{2 y-x}\right)^{2}, \quad e_{5 c}=\left(\frac{x}{2 z-x}\right)^{2}, \\
e_{6 c}=\frac{x^{2}}{y z-x^{2}}, \quad e_{7 c}=\frac{x^{2}(z-y)}{z\left(x^{2}+y z-2 x y\right)}, \quad e_{8 c}=\frac{x^{2}(z-y)}{y\left|x^{2}+y z-2 x z\right|}, \\
e_{9 c}=\frac{3 E_{1 c} D_{c}+\left|C_{c}\right|}{E_{1 c}^{3}}, \\
e_{10 c}=\frac{x(z-y)}{z(y-x)}, \quad e_{11 c}=\frac{x(z-y)}{y(z-x)}, \quad e_{12 c}=\frac{x}{y-x}, \\
e_{13 c}=\frac{x}{y}, \quad e_{14 c}=\frac{x}{z-x}, \quad e_{15 c}=\frac{x}{z},
\end{gathered}
$$

where

$$
\begin{gathered}
E_{1 c} \equiv 3 y z-2(y+z) x+x^{2}, \\
E_{2 c} \equiv(3 y z-(y+z) x)(y-x)(z-x), \\
E_{3 c} \equiv y z(y-x)^{2}(z-x)^{2}, \\
D_{c} \equiv E_{1 c}^{2}-3 E_{2 c}, \\
C_{c} \equiv 2 E_{1 c}^{3}-9 E_{1 c} E_{2 c}+27 E_{3 c},
\end{gathered}
$$

The series expansions of $R_{F}$ and $R_{D}$ using $u_{14 c}, v_{14 c}$, and $a_{14 c}$ are essentially the same as the third series expansion of $F(\varphi \mid m)$ and $E(\varphi \mid m)$ given in 38. It is interesting to see that $e_{5 c}, e_{14 c}$, and $e_{15 c}$ do not depend on $y$ nor on $m$. This was already pointed out for $e_{14 c}$ in 38 .

3.5. Minimum expansion factor. Again, in the complementary cases, we learn that the minimum expansion factor is achieved by $e_{1 c}$ or $e_{3 c}$ and the second minimum is done by $e_{3 c}$ and $e_{5 c}$. Based on the conclusion we reached in $\$ 3.2$, we focus on four choices to compute the elliptic integrals by series expansions: $\left(u_{3}, v_{3}, a_{3}\right)$, $\left(u_{5}, v_{5}, a_{5}\right),\left(u_{3 c}, v_{3 c}, a_{3 c}\right)$, and $\left(u_{5 c}, v_{5 c}, a_{5 c}\right)$.

Figure 3 shows the contour map of the minimum of the four expansion factors associated with the focused series expansions, $e_{3}, e_{5}, e_{3 c}$, and $e_{5 c}$, as a two-dimensional graph of $x / z$ and $m_{c} \equiv(y-x) /(z-x)$, both of which move in the unit interval $(0,1)$. The whole region is separated into four subregions where each of the four expansion factors becomes the minimum. Roughly speaking, the minimum is (1) 
Contour Map of Minimum Expansion Factor

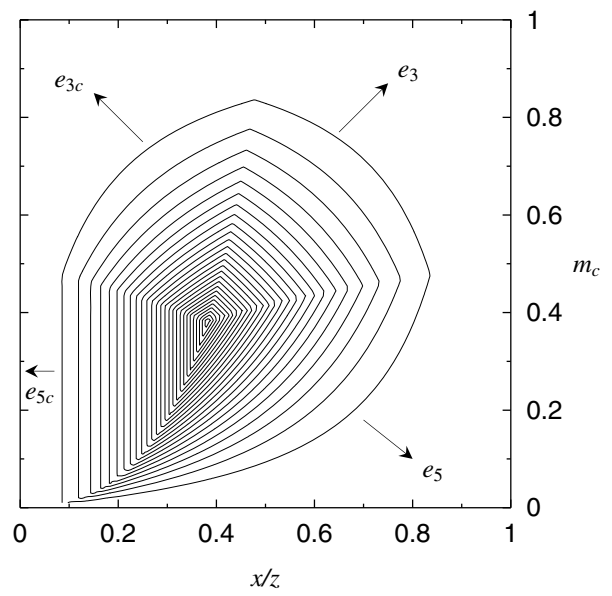

Figure 3. Contour map of minimum expansion factor. Illustrated are the contour map of the minimum of four expansion factors, $e_{3}, e_{5}, e_{3 c}$, and $e_{5 c}$, as two-dimensional graphs of $x / z$ and $m_{c} \equiv$ $(y-x) /(z-x)$. The contour level is started from 0 and incremented with a constant interval of $2 \times 10^{-3}$. The zero values are achieved at the boundary box. The minimum factor becomes (1) $e_{5}$ when $x / z$ is large and $m_{c}$ is small, (2) $e_{3}$ when $x / z$ and $m_{c}$ are large, (3) $e_{3 c}$ when $x / z$ is small and $m_{c}$ is large, and (4) $e_{5 c}$ when $x / z$ and $m_{c}$ are small. The central ridge separating the regions of $e_{3}$ and of $e_{5}$ and those of $e_{3 c}$ and of $e_{5 c}$ is expressed as the curve $m_{c}=[(x / z) /(1-x / z)]^{2}$. The right branch ridge discriminating the region of $e_{3}$ and that of $e_{5}$ is expressed as the curve $m_{c}=$ $\sqrt{x / z} /(1+\sqrt{x / z})$. Meanwhile, the left branch ridge dividing the region of $e_{3 c}$ and that of $e_{5 c}$ is expressed as the curve $m_{c}=(1-$ $x / z) /(2-x / z)$. The maximum value is achieved at the single intersection of these separatrices. It is $9-4 \sqrt{5} \sim 0.0557$ and reached when $x / z=m_{c}=(3-\sqrt{5}) / 2 \sim 0.382$.

$e_{5}$ when $x / z$ is large and $m_{c}$ is small, (2) $e_{3}$ when $x / z$ and $m_{c}$ are large, (3) $e_{3 c}$ when $x / z$ is small and $m_{c}$ is large, and (4) $e_{5 c}$ when $x / z$ and $m_{c}$ are small.

The separatrix between the ordinary pair $\left(e_{3}, e_{5}\right)$ and the complementary pair $\left(e_{3 c}, e_{5 c}\right)$ is the curve

$$
m_{c}=\left(\frac{x / z}{1-x / z}\right)^{2},
$$

which is equivalent with the condition

$$
x(y+z)=y z .
$$

This expression is obtained by solving the conditions $e_{3}=e_{3 c}$ and $/$ or $e_{5}=e_{5 c}$, the results of which coincide with each other. At any rate, we select the ordinary pair if $x(y+z)>y z$ and the complementary pair otherwise. 
Meanwhile, the separatrix between $e_{3}$ and $e_{5}$ is the curve

$$
m_{c}=\frac{\sqrt{x / z}}{1+\sqrt{x / z}},
$$

which is translated as

$$
x\left(y^{2}+z^{2}\right)=z\left(x^{2}+y^{2}\right) .
$$

Thus, if $x\left(y^{2}+z^{2}\right)<z\left(x^{2}+y^{2}\right)$, then we choose $\left(u_{3}, v_{3}, a_{3}\right)$ else $\left(u_{5}, v_{5}, a_{5}\right)$. A similar separatrix between $e_{3 c}$ and $e_{5 c}$ is the curve

$$
m_{c}=\frac{1-x / z}{2-x / z},
$$

which is rewritten as

$$
2 y z=z^{2}+x y \text {. }
$$

Therefore, we use $\left(u_{3 c}, v_{3 c}, a_{3 c}\right)$ when $2 y z<z^{2}+x y$ and $\left(u_{5 c}, v_{5 c}, a_{5 c}\right)$ otherwise.

The minimum expansion factor has a single maximum at the single intersection of the three separatrices. The coordinates of the cross point are solved as

$$
(x / z)_{\mathrm{MAX}}=\left(m_{c}\right)_{\mathrm{MAX}}=\frac{3-\sqrt{5}}{2}=0.3819660 \ldots
$$

This means that

$$
(y / z)_{\mathrm{MAX}}=1-(x / z)_{\mathrm{MAX}}=\frac{\sqrt{5}-1}{2}=0.6180340 \ldots
$$

and associates with the value of the expansion factor

$$
e_{\mathrm{MAX}}=9-4 \sqrt{5}=0.05572809 \ldots
$$

Since $(0.0557)^{6} \sim 3.0 \times 10^{-8},(0.0557)^{13} \sim 5.0 \times 10^{-17}$, and $(0.0557)^{28} \sim 9.6 \times 10^{-35}$, we anticipate that at most 6,13 , and 28 terms of the series expansion are enough for the single, double, and quadruple precision computations.

A similar discussion can be applied to the quartet of $e_{1}, e_{3}, e_{1 c}$, and $e_{3 c}$ which we did not adopt. The maximum value of the minimum expansion factor becomes around 0.0317 in this case. See Figure 4. This time $(0.0317)^{5} \sim 3.2 \times 10^{-8}$, $(0.0317)^{11} \sim 3.2 \times 10^{-17}$, and $(0.0317)^{23} \sim 3.3 \times 10^{-35}$. Then the expected numbers of terms reduce to 5,11 , and 23 , respectively. Nevertheless, the degree of reduction is not large enough to compensate the complexity to evaluate the auxiliary integrals in substantially two-dimensional arrays faced with the series expansions using $\left(u_{1}, v_{1}, a_{1}\right)$ or $\left(u_{1 c}, v_{1 c}, a_{1 c}\right)$.

At any rate, it is noteworthy that the so-called critical region where $x / z$ and $y / z$ are small is well covered by the combination of the series expansions using $\left(u_{5}, v_{5}, a_{5}\right)$ and its complement; rather, most difficult is the case of middle values where $x / z \sim 0.38$ and $y / z \sim 0.62$.

3.6. Complete integrals. Let us consider how to compute the complete integrals by the present formulation. For this purpose, we use the following formulas derived 


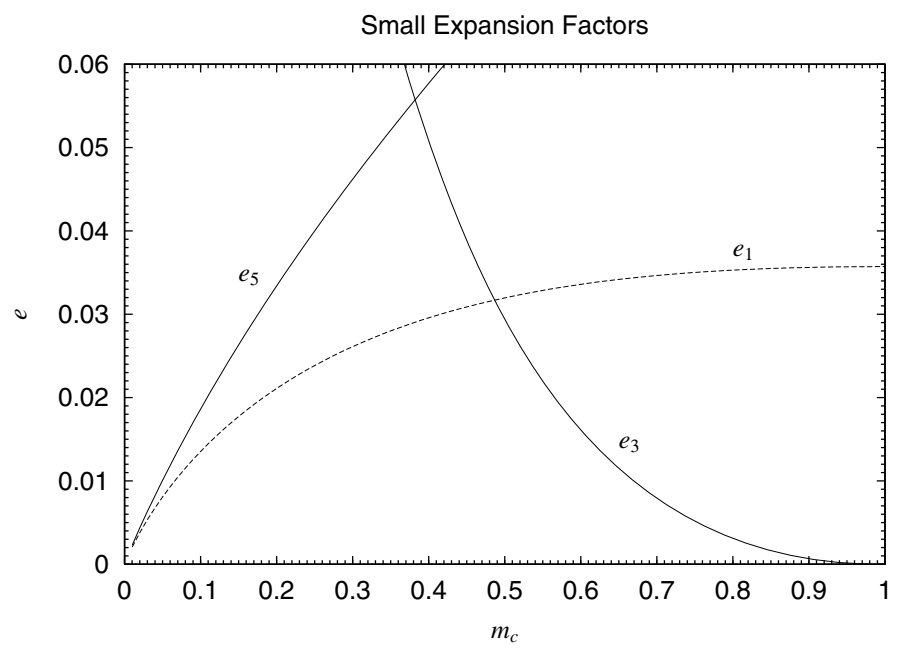

Figure 4. Small expansion factors along the main separatrix. Shown are the curve of small expansion factors of the series expansions along the main separatrix $x(y+z)=y z$ where $e_{1}=e_{1 c}$, $e_{3}=e_{3 c}$, and $e_{5}=e_{5 c}$. The curves are plotted as functions of $m_{c} \equiv(y-x) /(z-x)$. The crossing of the curve of $e_{1}$ and that of $e_{3}$ occurs at $\left(m_{c}, e\right) \sim(0.4868,0.0317)$ while that of the curve of $e_{3}$ and that of $e_{5}$ locates at $\left(m_{c}, e\right) \sim(0.3820,0.0557)$

from the special addition formulas:

$$
\begin{gathered}
R_{F}(0, y, z)=2 R_{F}\left(x_{m}, y_{m}, z_{m}\right) \\
R_{D}(0, y, z)=2 R_{D}\left(x_{m}, y_{m}, z_{m}\right)+\frac{3}{z_{m} \sqrt{z}} \\
R_{J}(0, y, z, p)=2 R_{J}\left(x_{m}, y_{m}, z_{m}, p_{m}\right)+\left(\frac{3}{\sqrt{p}}\right) R_{C}\left(p\left(y_{m}+z_{m}\right), p_{m}^{2}\right),
\end{gathered}
$$

where

$$
x_{m} \equiv \sqrt{y z}, y_{m} \equiv y+x_{m}, \quad z_{m} \equiv z+x_{m}, \quad p_{m} \equiv p+x_{m},
$$

are the modified parameters for complete integrals satisfying the order condition

$$
0 \leq x_{m} \leq y_{m} \leq z_{m} .
$$

Refer to Appendix B.3 for the derivation of the above formulas. They are also special cases of the duplication theorems presented in $\$ 19.26$ (iii) of [35]. Their translation into classic forms become

$$
\begin{gathered}
K(m)=2 F\left(\varphi_{m} \mid m\right), \\
E(m)=2 E\left(\varphi_{m} \mid m\right)-\left(\frac{m}{1+k_{c}}\right), \\
\Pi(n \mid m)=2 \Pi\left(\varphi_{m}, n \mid m\right)+\left(\frac{n}{\sqrt{n_{c}}}\right) R_{C}\left(n_{c}\left(1+k_{c}\right)^{2},\left(n_{c}+k_{c}\right)^{2}\right),
\end{gathered}
$$


where

$$
k_{c} \equiv \sqrt{m_{c}}=\sqrt{1-m},
$$

is the complementary modulus and

$$
\varphi_{m} \equiv \tan ^{-1}\left(\frac{1}{\sqrt{k_{c}}}\right)
$$

is the amplitude corresponding to the modified parameters.

The modified parameters, $x_{m}, y_{m}$, and $z_{m}$, satisfy the condition to locate on the main separatrix shown in Figure 3 as

$$
\frac{y_{m}-x_{m}}{z_{m}-x_{m}}-\left(\frac{x_{m}}{z_{m}-x_{m}}\right)^{2}=\frac{y}{z}-\left(\frac{\sqrt{y z}}{z}\right)^{2}=0 .
$$

Then the computation of the complete integrals can be performed by the series expansions based on $\left(u_{3}, v_{3}, a_{3}\right)$ or on $\left(u_{5}, v_{5}, a_{5}\right)$ using the modified parameters.

3.7. Algorithm. Summarizing the results in the previous subsections, we present here algorithms to compute the three elliptic integrals $R_{F}(x, y, z), R_{D}(x, y, z)$, and $R_{J}(x, y, z, p)$ simultaneously. The reason why we compute them simultaneously is that the core part of three procedures is the same, and therefore can be shared in the simultaneous computation. Below, we assume that the input parameters are already ordered as $0 \leq x \leq y \leq z$.

First, the complete integrals where $x=0$ are computed from the given $y, z$, and $p$ by the following algorithm:

C1. Compute $x_{m}, y_{m}, z_{m}$, and $p_{m}$ from $y, z$, and $p$ by equation (3.29).

C2. If $x_{m}>(x / z)_{\text {MAX }} z_{m}$ where $(x / z)_{\text {MAX }}$ is given in equation (3.23), then compute $u_{3}, v_{3}$, and $a_{3}$ from $x_{m}, y_{m}$, and $z_{m}$ by equation (2.35). Else compute $u_{5}, v_{5}$, and $a_{5}$ from $x_{m}, y_{m}$, and $z_{m}$ by equation (2.37).

C3. Evaluate equations (2.31) through (2.33) using $z_{m}, p_{m}$, and thus computed $u_{3}, v_{3}$, and $a_{3}$ or $u_{5}, v_{5}$, and $a_{5}$.

C4. Compute $R_{C}\left(p\left(y_{m}+z_{m}\right), p_{m}^{2}\right)$ and obtain the complete integrals from the evaluated incomplete integrals by equations (3.26) through (3.28).

Next, the incomplete integrals are computed from the given $x, y, z$, and $p$ as follows:

IC1. If $x(y+z)<y z$, then compute $x_{c}, y_{c}, z_{c}$, and $p_{c}$ from $x, y, z$, and $p$ by equation (3.8) and copy them to the working parameters $x_{w}, y_{w}, z_{w}$, and $p_{w}$. Else copy $x, y, z$, and $p$ to the working parameters.

IC2. If $x_{w}\left(y_{w}^{2}+z_{w}^{2}\right)<z_{w}\left(x_{w}^{2}+y_{w}^{2}\right)$, then compute $u_{3}, v_{3}$, and $a_{3}$ from $x_{w}, y_{w}$, and $z_{w}$ by equation (2.35). Else compute $u_{5}, v_{5}$, and $a_{5}$ from $x_{w}, y_{w}$, and $z_{w}$ by equation (2.37).

IC3. Evaluate equations (2.31) through (2.33) using $z_{w}, p_{w}$, and thus computed $u_{3}, v_{3}$, and $a_{3}$ or $u_{5}, v_{5}$, and $a_{5}$.

IC4. If $x, y, z$, and $p$ are used as the working parameters, return the evaluated integrals. Else compute the associated complete integrals $R_{F}(0, y-x, z-x)$, $R_{D}(0, y-x, z-x)$, and $R_{J}(0, y-x, z-x, p-x)$ by calling the routine of complete integrals, compute $R_{C}\left(y z(p-x), p p_{c}\right)$, and transform the evaluated integrals by the formulas given as equations (3.5) through (3.7).

The steps C2 and C3 are almost the same as IC2 and IC3, respectively.

In the above procedures, we need to compute the auxiliary integrals, $R_{C}(x, y)=$ $I_{01}(x, y), I_{0 k}(x, y)$ for $k \geq 2, I_{011}(x, y, z)$, and $I_{0 k 1}(x, y, z)$ for $k \geq 2$. They are 
efficiently evaluated by the formulas given in Appendix $\mathrm{A}$ as equations (A.20), (A.6), (A.12), and (A.13), respectively.

\section{Numerical EXAMPles}

In this section, we present the result of numerical examination on three points of the new series expansions. First, in 4.1 we confirm the correctness of our assumption on the nature of expansion factors in the worst case, namely at the peak of the contour map of the minimum expansion factor shown in Figure 3 , Next, in 4.2 , we observe the manner of convergence of the series expansions of the three elliptic integrals in this worst case. Finally, in 4.3. we compare the errors of the new series expansions near the logarithmic singularity with those of the existing asymptotic expansions summarized in Appendix C

4.1. Proximity of expansion factor. According to equation (2.9), the factors of the series expansions, $e$, are functions of the index $j$ as well as the integral $\operatorname{arguments} x, y, z$, and/or $p$. Also, they may depend on the kind of integrals, i.e., whether $R_{F}, R_{J}$, or $R_{D}$ in general. However, in discussing the rate of convergence of the series expansions in $\$ 2$, we assumed that the expansion factor remains to be constant independently with the index of terms and with the kind of integrals. This is only a conjecture. Thus, first of all, we must examine the degree to which this assumption is correct.

For this purpose, we numerically compute the ratio of adjacent terms of the series expansions of $R_{F}(x, y, z), R_{D}(x, y, z)$, and $R_{J}(x, y, z, p)$ by using the transformed parameters $\left(u_{3}, v_{3}, a_{3}\right)$ for the worst case, i.e., at the maximum peak in the contour map of the minimum expansion factor, Figure 3. For simplicity, we set $z=1$ and choose $p$ arbitrary. Then the input arguments become

$$
x=\frac{3-\sqrt{5}}{2}, y=\frac{-1+\sqrt{5}}{2}, z=1, \quad p=\frac{1}{2},
$$

where the last numerical value was arbitrary. These lead to the transformed parameters and the estimated expansion factor as

$$
u_{3}=\frac{3-\sqrt{5}}{2}, \quad v_{3}=\frac{1+\sqrt{5}}{4}, a_{3}=\frac{-7+3 \sqrt{5}}{8}, e_{3}=9-4 \sqrt{5}
$$

Our assumption in $₫ 2$ is that the expansion factor, $e$, is (1) independent on the index of terms in the series expansion, and (2) common to all three integrals. In order to examine the validity of this assumption, we prepared Figure 5 showing the ratio, to this assumed value $e_{3}$, of the actual magnitude ratios of the adjacent two terms in the series expansions adopted such as

$$
\begin{aligned}
r_{k} \equiv \mid\left\{\left(\begin{array}{c}
-1 / 2 \\
k
\end{array}\right) a_{3}^{k} I_{0,2 k+1}\left(u_{3}, v_{3}\right)\right\} /\{ & \left.\left(\begin{array}{l}
-1 / 2 \\
k-1
\end{array}\right) a_{3}^{k-1} I_{0,2 k-1}\left(u_{3}, v_{3}\right)\right\} \mid \\
& =\frac{(2 k-1)\left|a_{3}\right| I_{0,2 k+1}\left(u_{3}, v_{3}\right)}{2 k I_{0,2 k-1}\left(u_{3}, v_{3}\right)},
\end{aligned}
$$

for $R_{F}$. The figure indicates that all the actual ratios are smaller than the estimated expansion factor for the index up to 10 . This means that all three series converge a little faster than expected. 


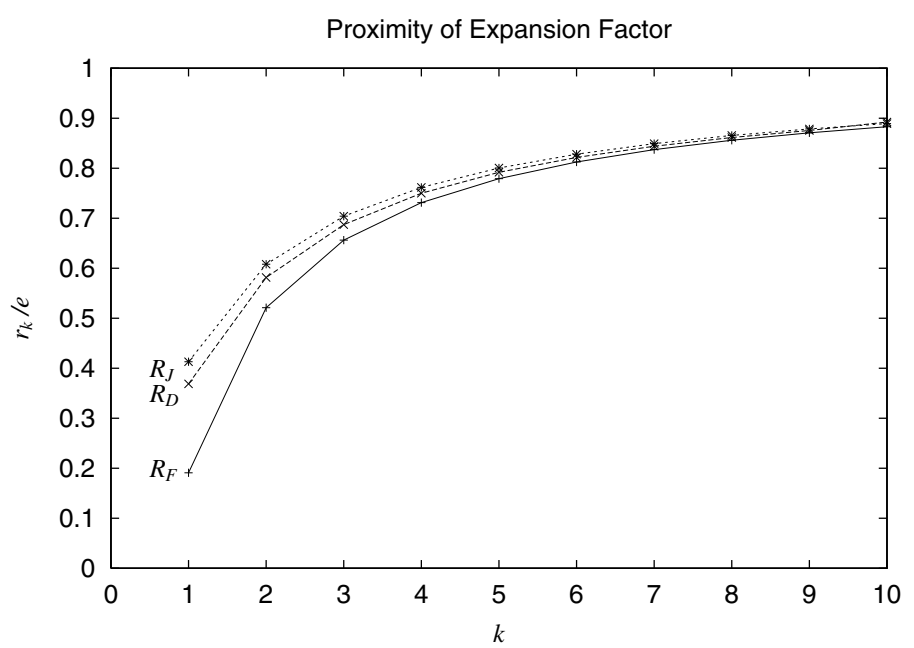

Figure 5. Proximity of expansion factor: worst case. Shown are the ratio of the magnitude ratio of the adjacent two terms in the series expansion to its estimate value. Considered is the worst case in the selected series expansion where $x \sim 0.382, y \sim 0.618$, $z=1$, and $p=1 / 2$. The series expansions are those using the linear remainder of the first type with the transformed parameters and the estimated expansion factor: $u_{3} \sim 0.382, v_{3} \sim 0.809$, and $a_{3} \sim-0.0365, e_{3} \sim 0.0557$.

4.2. Manner of convergence. Now that the assumption on the estimated expansion factor is positively confirmed in the previous subsection, we shall examine the rate of convergence of the series expansions we developed. As their representative, we again select the same worst case examined in the previous subsection. The numerical values of the integrals in double precision are computed by means of Carlson's duplication algorithms [9, 12, 37] as

$$
\begin{gathered}
R_{F}(x, y, z)=1.2526492359292611 \\
R_{D}(x, y, z)=1.4936381715116946 \\
R_{J}(x, y, z, p)=2.2867715735391876
\end{gathered}
$$

Table 1 lists the relative errors of the three integrals evaluated by the series expansion formulas given in equations (2.31) through (2.33) using the transformed parameters already described in the previous subsection. The relative errors decrease monotonically with respect to the number of terms. The measured errors coincide with those expected from the next term in the series expansion at the precision shown here. The table shows that the necessary minimum number of terms of the series expansions is 5 and 11 in the single and double precision environments, respectively. They are a little smaller than our previous estimate provided in 43.5 . This is because the actual magnitude of each term in the series decrease a little faster than expected as we observed in the previous subsection. 
TABLE 1. Manner of convergence: worst case. Illustrated are the relative errors of the truncated series expansions of three symmetric elliptic integrals, $R_{F}(x, y, z), R_{D}(x, y, z)$, and $R_{J}(x, y, z, p)$, where $x \sim 0.382, y \sim 0.618, z=1$, and $p=1 / 2$. The integral values are $R_{F} \sim 1.253, R_{D} \sim 1.494$, and $R_{J} \sim 2.287$.

\begin{tabular}{rrrr}
\hline$j$ & $\Delta R_{F} / R_{F}$ & $\Delta R_{D} / R_{D}$ & $\Delta R_{J} / R_{J}$ \\
\hline 0 & $6.5 \mathrm{E}-03$ & $1.3 \mathrm{E}-02$ & $1.4 \mathrm{E}-02$ \\
1 & $1.6 \mathrm{E}-04$ & $3.4 \mathrm{E}-04$ & $4.0 \mathrm{E}-04$ \\
2 & $5.2 \mathrm{E}-06$ & $1.2 \mathrm{E}-05$ & $1.4 \mathrm{E}-05$ \\
3 & $2.0 \mathrm{E}-07$ & $4.6 \mathrm{E}-07$ & $5.6 \mathrm{E}-07$ \\
4 & $8.1 \mathrm{E}-09$ & $1.9 \mathrm{E}-08$ & $2.3 \mathrm{E}-08$ \\
5 & $3.5 \mathrm{E}-10$ & $8.3 \mathrm{E}-10$ & $1.0 \mathrm{E}-09$ \\
6 & $1.6 \mathrm{E}-11$ & $3.8 \mathrm{E}-11$ & $4.7 \mathrm{E}-11$ \\
7 & $7.3 \mathrm{E}-13$ & $1.8 \mathrm{E}-12$ & $2.2 \mathrm{E}-12$ \\
8 & $3.4 \mathrm{E}-14$ & $8.2 \mathrm{E}-14$ & $1.1 \mathrm{E}-13$ \\
9 & $1.4 \mathrm{E}-15$ & $3.3 \mathrm{E}-15$ & $6.0 \mathrm{E}-15$ \\
\hline
\end{tabular}

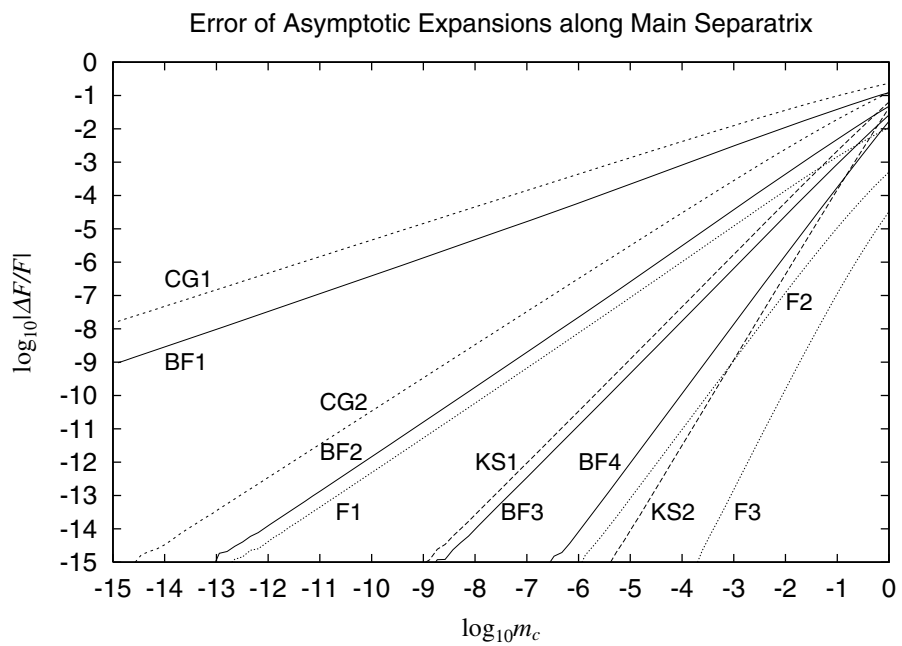

Figure 6. Relative errors of asymptotic expressions of $F(\varphi \mid m)$ along the main separatrix. Shown are the relative errors of the approximate expansions of $F(\varphi \mid m)$ along the main separatrix, where $\varphi=\tan ^{-1} \sqrt{1 /(1-m)}$. The errors are illustrated as functions of $m_{c}$ in a log-log manner.

4.3. Asymptotic expansions around logarithmic singularity. As we stated in $\$ 1$, the recent studies on the series expansion of elliptic integrals are focused on their asymptotic form in the so-called critical region where $x / z, y / z$, and/or $p / z$ are small. This condition is translated in terms of classic arguments as $\varphi \approx \pi / 2$, $m \approx 1$, and/or $n \approx 1$, and therefore $c \equiv \cos \varphi \approx 0, m_{c} \equiv 1-m \approx 0$, and/or $n_{c} \equiv 1-n \approx 0$. 
Let us take $F(\varphi \mid m)$ as an example. Its existing and new asymptotic expansions are summarized in Appendix C] They are coded as (1) BF1 through BF4 for the first through the fourth order classic expressions [2], (2) KS1 and KS2 for the first and the second order expressions of [29, (3) CG1 and CG2 for the first and the second order ones of [14, and (4) F1, F2, and F3 for the first, the second, and the third order ones of the new expressions using $\left(u_{5}, v_{5}, a_{5}\right)$ derived in $\$ 2.5$,

We do not show the results of those using $\left(u_{1}, v_{1}, a_{1}\right)$ here. This is partly because their expressions become complicated and partly because they become almost the same as those based on $\left(u_{5}, v_{5}, a_{5}\right)$ near the singularity. The superiority of the expansion using $\left(u_{1}, v_{1}, a_{1}\right)$ becomes eminent in the noncritical region such as when $m_{c}>10^{-3}$ along the main separatrix.

Figure 6 shows the relative errors of these approximate expressions along the main separatrix as functions of $m_{c}$ in a log-log manner. This is the worst case for the expressions other than CG1 and CG2. The errors illustrated in the figure are roughly ordered as

$$
\begin{aligned}
\left|\Delta F_{\mathrm{F} 3}\right| & \ll\left|\Delta F_{\mathrm{KS} 2}\right| \ll\left|\Delta F_{\mathrm{F} 2}\right|<\left|\Delta F_{\mathrm{BF} 4}\right| \ll\left|\Delta F_{\mathrm{BF} 3}\right|<\left|\Delta F_{\mathrm{KS} 1}\right| \\
& \ll\left|\Delta F_{\mathrm{F} 1}\right|<\left|\Delta F_{\mathrm{BF} 2}\right|<\left|\Delta F_{\mathrm{CG} 2}\right| \ll\left|\Delta F_{\mathrm{BF} 1}\right|<\left|\Delta F_{\mathrm{CG} 1}\right|,
\end{aligned}
$$

near the logarithmic singularity $m_{c}=c=0$. Figure 6 illustrates the result only along the main separatrix where $m_{c} \tan ^{2} \varphi=1$, and therefore

$$
c=c_{\text {crit }} \equiv \sqrt{\frac{k_{c}}{1+k_{c}}} .
$$

We observe the same tendency when $c$ is smaller than this critical value while the error magnitude decreases significantly when $c / c_{\text {crit }} \rightarrow 0$. If $c>c_{\text {crit }}$, on the other hand, the complementary expressions show the similar inequalities.

The great inequality sign, $\ll$, in equation (4.7) indicates that the power law index with respect to $k_{c} \equiv \sqrt{m_{c}}$ is different before and after the sign. For example, $F_{\mathrm{CG} 2}, F_{\mathrm{BF} 2}$, and $F_{\mathrm{F} 1}$ are correct up to the order of $k_{c}$. Meanwhile, $F_{\mathrm{KS} 1}$ and $F_{\mathrm{BF} 3}$ are so up to the order of $k_{c}^{2}$. As for the magnitude of proportional coefficients of the leading error term among the asymptotic expressions of the same power law index, we confirm that the new ones are the least, the classic ones are the second least, then come those of Karp and Sitnik, and those of Carlson and Gustafson.

Noteworthy is the smallness of the error constants of the new asymptotic expressions in the noncritical region, say when $m_{c}>10^{-3}$ along the main separatrix. See Figure 6 again. As a result, some of the new expressions become better than those of the higher power law index for the purpose of low precision approximation. For example, the error of $F_{\mathrm{F} 2}$ of the order of $k_{c}^{4}$ is smaller than that of $F_{\mathrm{KS} 2}$ of the order of $k_{c}^{5}$ in the single precision environment, namely when $|\Delta F / F| \geq 10^{-7}$.

\section{Conclusion}

We discussed a general formulation to construct the series expansions of an elliptic integral by using the binomial expansion of the reciprocal square root in \$2. In the case of Carlson's standard symmetric elliptic integrals, $R_{F}(x, y, z)$, $R_{D}(x, y, z)$, and $R_{J}(x, y, z, p)$, we split the given cubic polynomial in the square root, $P(t)=(t+x)(t+y)(t+z)$, into its main part, $P_{0}(t)$, and the remainder, $\Delta P(t)$, in several ways. Then we developed 15 kinds of their series expansions. Four of them are essentially the same as the classic expansions given in the literature. Meanwhile the other eleven are new. 
Next, in $\$ 3$, by utilizing the special addition formulas of the integrals, we obtained the complementary series expansions for each of the above 15 ones. Thus we have 30 different series expansions in total for each of $R_{F}(x, y, z), R_{D}(x, y, z)$, and $R_{J}(x, y, z, p)$. Then we select four of them as the best formulas after considering the balance between the rate of convergence and the amount of computational labor.

They are the series expansions derived from the splitting with the linear remainder of the first type case A such that $P_{0}(t)=(t+u)(t+v)^{2}$ and $\Delta P(t)=a(t+u)$ as

$$
\begin{gathered}
R_{F}(x, y, z)=\sum_{j=0}^{\infty}\left(\begin{array}{c}
-1 / 2 \\
j
\end{array}\right) a^{j} I_{0,2 j+1}(u, v) \\
=I_{01}(u, v)-\frac{a}{2} I_{03}(u, v)+\frac{3 a^{2}}{8} I_{05}(u, v)-\cdots, \\
R_{D}(x, y, z)=3 \sum_{j=0}^{\infty}\left(\begin{array}{c}
-1 / 2 \\
j
\end{array}\right) a^{j} I_{0,2 j+1,1}(u, v, z) \\
=3 I_{011}(u, v, z)-\frac{3 a}{2} I_{031}(u, v, z)+\frac{9 a^{2}}{8} I_{051}(u, v, z)-\cdots, \\
R_{J}(x, y, z, p)=3 \sum_{j=0}^{\infty}\left(\begin{array}{c}
-1 / 2 \\
j
\end{array}\right) a^{j} I_{0,2 j+1,1}(u, v, p) \\
=3 I_{011}(u, v, p)-\frac{3 a}{2} I_{031}(u, v, p)+\frac{9 a^{2}}{8} I_{051}(u, v, p)-\cdots,
\end{gathered}
$$

where $I_{j k}(x, y)$ and $I_{j k \ell}(x, y, z)$ are the auxiliary symmetric integrals we introduced. The auxiliary integrals are all elementary functions as explained in Appendix A where we provided practical procedures to compute them.

Here $u, v$, and $a$ are the newly introduced parameters. They are uniquely transformed from the input parameters $x, y$, and $z$ and have no relations with $p$. The transformed parameters of the selected four series expansions are explicitly expressed as functions of $x, y$, and $z$ as

$$
\begin{aligned}
& \left(u_{3}, v_{3}, a_{3}\right)=\left(x, \frac{y+z}{2}, \frac{-(z-y)^{2}}{4}\right), \\
& \left(u_{5}, v_{5}, a_{5}\right)=\left(z, \frac{x+y}{2}, \frac{-(y-x)^{2}}{4}\right),
\end{aligned}
$$

and their complements

$$
\begin{gathered}
\left(u_{3 c}, v_{3 c}, a_{3 c}\right)=\left((y-x)(z-x), y z-\frac{x(y+z)}{2}, \frac{-x^{2}(z-y)^{2}}{4}\right), \\
\left(u_{5 c}, v_{5 c}, a_{5 c}\right)=\left(y(z-x),(y-x)\left(z-\frac{x}{2}\right), \frac{-x^{2}(y-x)^{2}}{4}\right) .
\end{gathered}
$$

Thus we evaluate the elliptic integrals by choosing one of these four series expansions giving the least errors. By using the approximate forms of the expansion factors, we analytically specified its rule of selection in a simple way as described in 33.7

Also, we obtained the series expansions of the complete integrals where $x=0$ by applying these formulations to the variation of duplication formulas which express the complete integrals in terms of the incomplete ones with the modified parameters $x_{m}=\sqrt{y z}, y_{m}=y+x_{m}, z_{m}=z+x_{m}$, and $p_{m}=p+x_{m}$. Thus the formulation has 
been completed within the framework of the series expansions. As a by-product, we obtained an efficient asymptotic expansion of the integrals around their logarithmic singularities using the transformed parameter sets, $\left(u_{5}, v_{5}, a_{5}\right)$ or $\left(u_{5 c}, v_{5 c}, a_{5 c}\right)$ as illustrated in Appendix C.4.

Then, we conducted some numerical experiments in order to confirm the effectiveness of the new series expansions obtained in \$4. Table 1 indicates that at most 5 and 11 terms of the suitably chosen series expansions are sufficient to evaluate the integrals in the single and double precision environments, respectively. This is the same for the case of complete integrals.

Finally, we should stress that the development of new series expansions greatly owes to the symmetric nature of Carlson's elliptic integrals. Indeed, the series expansions based on the transformed parameters mixing $\cos ^{2} \varphi \equiv x / z$ and $m \equiv$ $(z-y) /(z-x)$ in a complicated manner like $\left(u_{1}, v_{1}, a_{1}\right)$ would not be hinted from the classic expressions of the integrals treating $\varphi$ and $m$ in an asymmetric manner.

\section{APPENDIX}

\section{Appendix A. Computation of Auxiliary Symmetric integrals}

Let us describe how to compute efficiently the auxiliary symmetric integrals:

$$
\begin{gathered}
I_{j}(x) \equiv \frac{1}{2} \int_{0}^{\infty} \frac{d t}{(t+x)^{j} \sqrt{t+x}} \\
I_{j k}(x, y) \equiv \frac{1}{2} \int_{0}^{\infty} \frac{d t}{(t+x)^{j}(t+y)^{k} \sqrt{t+x}} \\
I_{j k \ell}(x, y, z) \equiv \frac{1}{2} \int_{0}^{\infty} \frac{d t}{(t+x)^{j}(t+y)^{k}(t+z)^{\ell} \sqrt{t+x}},
\end{gathered}
$$

where $j$ is a nonnegative integer and $k$ and $\ell$ are positive integers. These integrals are all elementary functions. Note that $I_{0}(x)$ is not needed for our calculations.

A.1. Direct expressions. Some integrals are directly expressed in terms of elementary functions as

$$
\begin{gathered}
I_{j}(x)=\frac{1}{(2 j-1) x^{j-1} \sqrt{x}}, \quad(j=1,2, \cdots) \\
I_{01}(x, y)=R_{C}(x, y)= \begin{cases}\frac{1}{\sqrt{x-y}} \log \left(\frac{\sqrt{x}+\sqrt{x-y}}{\sqrt{y}}\right) & (x>y), \\
\frac{1}{\sqrt{x}} & (x=y), \\
\frac{1}{\sqrt{y-x}} \tan ^{-1} \sqrt{\frac{y-x}{x}} & (x<y) .\end{cases}
\end{gathered}
$$


A.2. Forward recurrence formulas. The remaining integrals are efficiently computed by the forward recurrence formulas when the arguments are sufficiently different from each other:

$$
I_{j k \ell}(x, y, z)=\frac{I_{j, k, \ell-1}(x, y, z)-I_{j, k-1, \ell}(x, y, z)}{z-y}, \quad(j=0,1, \cdots ; k, \ell=1,2, \cdots),
$$

$$
I_{j k \ell}(x, y, z)=\frac{I_{j, k, \ell-1}(x, y, z)-I_{j-1, k, \ell}(x, y, z)}{z-x}, \quad(j, \ell=1,2, \cdots ; k=0,1, \cdots),
$$

where

$$
I_{j 0}(x, y)=I_{j}(x), \quad I_{j k 0}(x, y, z)=I_{j k}(x, y), \quad I_{j 0 \ell}(x, y, z)=I_{j \ell}(x, z) .
$$

For example, we obtain $I_{11}(x, y)$ and $I_{0 k 1}(x, y, z)$ as

$$
\begin{gathered}
I_{11}(x, y)=\frac{I_{1}(x)-I_{01}(x, y)}{y-x}=\frac{1}{y-x}\left(\frac{1}{\sqrt{x}}-R_{C}(x, y)\right), \\
I_{011}(x, y, z)=\frac{I_{01}(x, y)-I_{01}(x, z)}{z-y}=-\left(\frac{R_{C}(x, z)-R_{C}(x, y)}{z-y}\right), \\
I_{0 k 1}(x, y, z)=\frac{I_{0 k}(x, y)-I_{0, k-1,1}(x, y, z)}{z-y}, \quad(k=2,3, \cdots)
\end{gathered}
$$

where we used the direct expression of $I_{j}(x)$ given in equation (A.4).

The last two formulas of $I_{j k \ell}(x, y, z)$, equations (A.8) and (A.9), are redundant in some cases. In order to minimize the information loss, it is better to select the formula with the larger denominator in redundant cases. Thus we choose equation (A.8) when $|z-y|>|z-x|$ and equation (A.9) otherwise.

A.3. Series expansions. The forward recurrence formulas suffer cancellation problems when some of the parameters are similar. These are overcome by using the series expansions. For this purpose, we consider the following binomial expansion:

$$
\frac{1}{(t+y)^{k}}=\frac{1}{[(t+x)+(y-x)]^{k}}=\frac{1}{(t+x)^{k}} \sum_{q=0}^{\infty}\left(\begin{array}{c}
k+q-1 \\
q
\end{array}\right)\left(\frac{x-y}{t+x}\right)^{q} .
$$

Thus the integrals containing the factor $1 /(t+y)^{k}$ can be expanded when $x \approx y$ as

$$
\begin{gathered}
I_{j k}(x, y)=\sum_{q=0}^{\infty}\left(\begin{array}{c}
k+q-1 \\
q
\end{array}\right)(x-y)^{q} I_{j+k+q}(x), \\
I_{j k \ell}(x, y, z)=\sum_{q=0}^{\infty}\left(\begin{array}{c}
k+q-1 \\
q
\end{array}\right)(x-y)^{q} I_{j+k+q, \ell}(x, z) .
\end{gathered}
$$


Similarly, we obtain the expansions of $I_{j k \ell}(x, y, z)$ as

$$
I_{j k \ell}(x, y, z)=\sum_{q=0}^{\infty}\left(\begin{array}{c}
\ell+q-1 \\
q
\end{array}\right)(x-z)^{q} I_{j+\ell+q, k}(x, y),
$$

when $|z-x| \ll x$,

$$
I_{j k \ell}(x, y, z)=\sum_{q=0}^{\infty}\left(\begin{array}{c}
\ell+q-1 \\
q
\end{array}\right)(y-z)^{q} I_{j, k+\ell+q}(x, y),
$$

when $|z-y| \ll y$, and

$I_{j k \ell}(x, y, z)=\sum_{q=0}^{\infty} \sum_{r=0}^{q}\left(\begin{array}{c}k+q-r-1 \\ q-r\end{array}\right)\left(\begin{array}{c}\ell+r-1 \\ r\end{array}\right)(x-y)^{q-r}(x-z)^{r} I_{j+k+\ell+q}(x)$,

when $|x-y| \ll x$ and $|x-z| \ll x$.

As for the practical judgment condition to switch from the forward recurrence formula to the series expansion, we recommend setting the relative smallness as $1 / 8$. For example, we apply the series expansion of $I_{j k}(x, y)$ when $|x-y|<x / 8$.

A.4. Practical computation of $R_{C}(x, y)$. The first expansion formula, equation (A.15), includes the case of $R_{C}(x, y)=I_{01}(x, y)$. Then we use the following expression for the practical calculation of $R_{C}(x, y)$ :

$$
R_{C}(x, y)= \begin{cases}\frac{1}{\sqrt{x-y}} \log \left(\frac{\sqrt{x}+\sqrt{x-y}}{\sqrt{y}}\right) & (y \leq(7 / 8) x), \\ \frac{1}{\sqrt{y-x}} \tan ^{-1} \sqrt{\frac{y-x}{x}} & (y \geq(9 / 8) x), \\ \frac{1}{\sqrt{x}} \sum_{q=0}^{Q} \frac{1}{2 q+1}\left(\frac{x-y}{x}\right)^{q} & \text { (otherwise). }\end{cases}
$$

The number of terms in the above series expansion $Q$ must be 6,15 , and 35 in the single, double, and quadruple precision environments, respectively, in order to assure that the truncation errors are less than the machine epsilon. By comparing with the quadruple precision computations, we confirmed that these critical values are correct in the single and double precision environments for $R_{C}(x, y)$.

This combination of the direct approach and the series expansion method to compute $R_{C}(x, y)$ is around three times faster than Carlson's duplication method 11, 12. See Table 2, It compares the CPU times of these two methods to compute $R_{C}(x, y)$. The CPU times are measured at a $\mathrm{PC}$ with the Intel Core Duo processor, averaged for $x$ and $y$ in the unit box $(0,1) \times(0,1)$, and scaled by that to compute the double precision sine function.

A.5. Backward recurrence formulas. The series expansion methods are generally much slower than the direct computation method or its combination with the forward recurrence formulas. An alternative solution to the cancellation problem is the usage of backward recurrence formulas:

$$
\begin{aligned}
& I_{0 k}(x, y)=\frac{1}{2 k-1}\left(2 k(y-x) I_{0, k+1}(x, y)+\frac{\sqrt{x}}{y^{k}}\right), \quad(k=1,2, \cdots), \\
& I_{j k}(x, y)=I_{j-1, k+1}(x, y)+(y-x) I_{j, k+1}(x, y), \quad(j, k=1,2, \cdots), \\
& I_{j k \ell}(x, y, z)=I_{j, k+1, \ell-1}(x, y, z)-(z-y) I_{j, k+1, \ell}(x, y, z), \\
& I_{j k \ell}(x, y, z)=I_{j+1, k, \ell-1}(x, y, z)-(z-x) I_{j+1, k, \ell}(x, y, z) .
\end{aligned}
$$


TABle 2. Averaged CPU times to compute $R_{C}(x, y)$. Listed are the CPU times of two methods to compute $R_{C}(x, y)$ in the single and double precision environments: the duplication method described in 12 and the combined formula of the direct expressions and the series expansion explained in equation (A.20) in Appendix A.4. The CPU times are averaged for $x$ and $y$ in the unit box $(0,1) \times(0,1)$. The necessary minimum numbers of the terms of the series are 6 and 15 in the single and double precision computations. The unit of CPU time is that to compute the double precision sine function.

\begin{tabular}{llrr}
\hline Method & Reference & Single & Double \\
\hline Duplication & {$[11,[12$} & 6.90 & 7.44 \\
Direct Expression + Series Expansion & This article & 2.36 & 2.91 \\
\hline
\end{tabular}

Again, the index $j$ is a nonnegative integer and $k$ and $\ell$ are positive integers in the last two formulas. In order to use these recurrence formulas, one must prepare the integrals for some large indices as the starting values of the backward recurrence.

A.6. Sample usage of backward recurrence formulas. As an illustration to use the backward recurrence formulas, we describe how to compute efficiently a set of $I_{0 j}(x, y)$ for $j=1,2, \cdots, J$ when $x \approx y$. In this case, we first evaluate the integral with the largest index by a truncation of equation (A.15) as

$$
I_{0 J}(x, y)=\frac{1}{x^{J-1} \sqrt{x}} \sum_{q=0}^{Q} \frac{1}{2 J+2 q-1}\left(\frac{x-y}{x}\right)^{q},
$$

where $Q$ is the truncation index. If $|x-y|<x / 8$, the necessary minimum of $Q$ to assure the machine epsilon accuracy is at most 6,15 , and 35 in the single, double, and quadruple precision environments, respectively. In order to minimize the round-off errors, the summation should be done in the decreasing order with respect to $q$. Finally, we deploy equation A.21 in order to obtain the remaining integrals $I_{0 j}(x, y)$ in the reverse order from $j=J-1$ down to $j=1$.

As another example, let us calculate a set of $I_{0 j 1}(x, y, z)$ for $j=1,2, \cdots, J$ when $y \approx z$. In this case, we first fix a certain large number $Q$ and prepare $I_{0 j}(x, y)$ for $j=1,2, \cdots, J+Q+1$. The preparation is done by the combination of the series expansions and the backward recurrence formulas as described in the above if $x \approx y$, say $|x-y|<x / 8$. Otherwise we compute $I_{01}(x, y)$ by equation (A.5) and $I_{0 j}(x, y)$ for $j=2,3, \cdots, J+Q+1$ by using equation (A.6). Second, we evaluate the integral with the largest index by a truncation of equation (A.16) as

$$
I_{0 J 1}(x, y, z)=\sum_{\ell=0}^{Q}(y-z)^{q} I_{0, J+q+1}(x, y) .
$$

Finally, we obtain the remaining integrals $I_{0 j 1}(x, y, z)$ for $j=1,2, \cdots, J-1$ by using equation (A.23) as

$$
I_{0 j 1}(x, y, z)=I_{0, j+1}(x, y)+(y-z) I_{0, j+1,1}(x, y, z),
$$

in the reverse order of $j$. 


\section{Appendix B. Derivation of Special addition formulas}

Let us derive the special addition formulas of the symmetric elliptic integrals and their variations quoted in the main text from their general addition theorems.

B.1. General addition theorems. The general addition theorems of the symmetric elliptic integrals are found as the formulas 19.26.1, 19.26.7, and 19.26.9 of 35] as

$$
\begin{gathered}
R_{F}(x+\lambda, y+\lambda, z+\lambda)+R_{F}(x+\mu, y+\mu, z+\mu)=R_{F}(x, y, z), \\
R_{D}(x+\lambda, y+\lambda, z+\lambda)+R_{D}(x+\mu, y+\mu, z+\mu) \\
=R_{D}(x, y, z)-\left(\frac{3}{\sqrt{z(z+\lambda)(z+\mu)}}\right) \\
R_{J}(x+\lambda, y+\lambda, z+\lambda, p+\lambda)+R_{J}(x+\mu, y+\mu, z+\mu, p+\lambda) \\
=R_{J}(x, y, z, p)-3 R_{C}(\gamma-\delta, \gamma),
\end{gathered}
$$

where: (1) $x$ and $y$ are nonnegative, (2) $z$ and $\lambda$ are positive, (3) $\mu$ is uniquely determined from $x, y, z$, and $\lambda$ as

$$
\mu \equiv\left(\frac{\sqrt{x y z}+\sqrt{(x+\lambda)(y+\lambda)(z+\lambda)}}{\lambda}\right)^{2}-\lambda-(x+y+z),
$$

as given as the formula 19.26 .5 of [35], and (4) $\gamma$ and $\delta$ are cubic polynomials of $p$ defined as

$$
\begin{gathered}
\gamma \equiv p(p+\lambda)(p+\mu), \\
\delta \equiv(p-x)(p-y)(p-z),
\end{gathered}
$$

as given as the formula 19.26.10 of [35. The difference of $\gamma$ and $\delta$ is quadratic with respect to $p$ as

$$
\gamma-\delta=(\lambda+\mu+x+y+z) p^{2}+(\lambda \mu-x y-y z-z x) p+x y z .
$$

B.2. Special addition formulas. By setting $x=0$ and introducing the new variables as

$$
X \equiv \lambda, \quad Y \equiv y+\lambda, \quad Z \equiv z+\lambda, \quad P \equiv p+\lambda
$$

we rewrite $\mu, \gamma$, and $\gamma-\delta$ as

$$
\begin{gathered}
\mu=\frac{Y Z}{X}-X-(Y+Z-2 X)=\frac{X_{c}}{X} \\
\gamma=(P-X) P(P-X+\mu)=\frac{P P_{c}(P-X)}{X}, \\
\gamma-\delta=(\lambda+\mu+y+z) p^{2}+(\lambda \mu-y z) p \\
=\left(\frac{X_{c}}{X}+Y+Z-X\right)(P-X)^{2}=\frac{Y Z(P-X)^{2}}{X},
\end{gathered}
$$

where we introduced a pair of complementary parameters as

$$
X_{c} \equiv(Y-X)(Z-X), \quad P_{c} \equiv X(P-X)+(Y-X)(Z-X),
$$

and used a relation

$$
\lambda \mu=X_{c}=y z
$$


which holds when $x=0$. Then we obtain

$$
\begin{aligned}
& y+\mu=Y-X+\frac{X_{c}}{X}=\frac{Y_{c}}{X}, \quad z+\mu=Z-X+\frac{X_{c}}{X}=\frac{Z_{c}}{X}, \\
& p+\mu=P-X+\frac{X_{c}}{X}=\frac{P_{c}}{X},
\end{aligned}
$$

where

$$
Y_{c} \equiv Z(Y-X), \quad Z_{c} \equiv Y(Z-X),
$$

are another pair of complementary parameters. Using these expressions, we rewrite the general addition theorems as

$$
\begin{gathered}
R_{F}(X, Y, Z)=R_{F}(0, Y-X, Z-X)-R_{F}\left(\frac{X_{c}}{X}, \frac{Y_{c}}{X}, \frac{Z_{c}}{X}\right), \\
R_{D}(X, Y, Z)=R_{F}(0, Y-X, Z-X) \\
-R_{D}\left(\frac{X_{c}}{X}, \frac{Y_{c}}{X}, \frac{Z_{c}}{X}\right)-3 \sqrt{\frac{X}{(Z-X) Z Z_{c}}}, \\
R_{J}(X, Y, Z, P)=R_{J}(0, Y-X, Z-X, P-X) \\
-R_{J}\left(\frac{X_{c}}{X}, \frac{Y_{c}}{X}, \frac{Z_{c}}{X}, \frac{P_{c}}{X}\right)-3 R_{C}\left(\frac{Y Z(P-X)^{2}}{X}, \frac{P P_{c}(P-X)}{X}\right) .
\end{gathered}
$$

Utilizing the scalar multiplication formulas of the integrals

$$
\begin{array}{r}
R_{F}(r x, r y, r z)=\frac{R_{F}(x, y, z)}{\sqrt{r}}, R_{C}(r x, r y)=\frac{R_{C}(x, y)}{\sqrt{r}}, \\
R_{D}(r x, r y, r z)=\frac{R_{D}(x, y, z)}{r \sqrt{r}}, R_{J}(r x, r y, r z, r p)=\frac{R_{J}(x, y, z, p)}{r \sqrt{r}},
\end{array}
$$

given as the formulas 19.20.1 and 19.20.6 of [35], we further rewrite the above expressions of the integrals as

$$
\begin{gathered}
R_{F}(X, Y, Z)=R_{F}(0, Y-X, Z-X)-\sqrt{X} R_{F}\left(X_{c}, Y_{c}, Z_{c}\right) \\
R_{D}(X, Y, Z)=R_{D}(0, Y-X, Z-X)-X \sqrt{X} R_{D}\left(X_{c}, Y_{c}, Z_{c}\right) \\
-\left(\frac{3}{Z-X}\right) \sqrt{\frac{X}{Y Z}}, \\
R_{J}(X, Y, Z, P)=R_{J}(0, Y-X, Z-X, P-X)-X \sqrt{X} R_{J}\left(X_{c}, Y_{c}, Z_{c}, P_{c}\right) \\
-3 \sqrt{\frac{X}{P-X}} R_{C}\left(Y Z(P-X), P P_{c}\right) .
\end{gathered}
$$

These are the formulas to compute incomplete integrals from the complete and another incomplete ones. If we here rename $X, Y, Z$, and $P$ as $x, y, z$, and $p$, respectively, the formulas become the same as equations (3.5) through (3.7) given in the main text.

B.3. Variation of special addition formulas. In the special addition formulas, let us further assume that $\lambda=\mu$. Thanks to equation (B.4), the additional assumption is rewritten in terms of $\lambda, y$, and $z$ as

$$
\lambda=\frac{(\lambda+y)(\lambda+z)}{\lambda}-\lambda-(y+z) .
$$


This turns out to be a pure quadratic equation with respect to $\lambda$ and is solved as

$$
\lambda=\sqrt{y z},
$$

since $\lambda>0$. Refer to the discussion in $\S 19.26$ (ii) of [35].

Then the expressions of $\gamma-\delta$ and $\gamma$ change as

$$
\begin{gathered}
\gamma-\delta=p^{2}(2 \lambda+y+z)+p\left(\lambda^{2}-y z\right)=p^{2}(\sqrt{y}+\sqrt{z})^{2}, \\
\gamma=p(p+\lambda)^{2}=p(p+\sqrt{y z})^{2} .
\end{gathered}
$$

Substituting these expressions into the special addition formulas derived in the previous subsection, we obtain their variations to compute the complete integrals from the incomplete ones as

$$
\begin{gathered}
R_{F}(0, y, z)=2 R_{F}(\sqrt{y z}, y+\sqrt{y z}, z+\sqrt{y z}) \\
R_{D}(0, y, z)=2 R_{D}(\sqrt{y z}, y+\sqrt{y z}, z+\sqrt{y z})+\frac{3}{(z+\sqrt{y z}) \sqrt{z}} \\
R_{J}(0, y, z, p)=2 R_{J}(\sqrt{y z}, y+\sqrt{y z}, z+\sqrt{y z}, p+\sqrt{y z}) \\
+\left(\frac{3}{\sqrt{p}}\right) R_{C}\left(p(\sqrt{y}+\sqrt{z})^{2},(p+\sqrt{y z})^{2}\right)
\end{gathered}
$$

where we used the scalar multiplication theorem in rewriting $R_{C}$ in the last expression. By introducing the modified parameters as defined in equation (3.29), we arrive the same expressions as equations (3.26) through (3.28).

\section{Appendix C. Asymptotic expressions of $F(\varphi \mid m)$}

Let us summarize the existing and new asymptotic expansions of $F(\varphi \mid m)$ around its logarithmic singularity, $\varphi=\pi / 2$ and $m=1$. The expressions below are tagged as: (1) BF1 through BF4 for the classic expressions given in [2, (2) KS1 and KS2 for the expressions of [29, (3) CG1 and CG2 for those of [14, and (4) F1 through F3 for the new ones ones described in $\$ 2.5$.

C.1. Classic expressions. There exists a classic series expansion of the incomplete elliptic integral of the first and the second kinds with respect to $m_{c} \tan ^{2} \varphi$ as we noted in $\$ 2.8$. In case of $F(\varphi \mid m)$, its first four terms are reproduced from the formula 902.01 in [2] as

$$
\begin{gathered}
F_{\mathrm{BF} 1}=\log \left(\frac{1+s}{c}\right) \\
F_{\mathrm{BF} 2}=\left(1+\frac{m_{c}}{4}\right) \log \left(\frac{1+s}{c}\right)-\frac{m_{c} s}{4 c^{2}} \\
F_{\mathrm{BF} 3}=\left(1+\frac{m_{c}}{4}+\frac{9 m_{c}^{2}}{64}\right) \log \left(\frac{1+s}{c}\right)-\frac{m_{c} s}{4 c^{2}}\left(1+\frac{9 m_{c}}{16}\right)+\frac{3 m_{c}^{2} s}{32 c^{4}}, \\
F_{\mathrm{BF} 4}=\left(1+\frac{m_{c}}{4}+\frac{9 m_{c}^{2}}{64}+\frac{25 m_{c}^{3}}{256}\right) \log \left(\frac{1+s}{c}\right) \\
-\frac{m_{c} s}{4 c^{2}}\left(1+\frac{9 m_{c}}{16}+\frac{25 m_{c}^{2}}{256}\right)+\frac{3 m_{c}^{2} s^{3}}{32 c^{4}}\left(1+\frac{25 m_{c}}{36}\right)-\frac{5 m_{c}^{3} s^{5}}{96 c^{6}}
\end{gathered}
$$

where $m_{c} \equiv 1-m$ is the complementary parameter, and

$$
c \equiv \cos \varphi, \quad s \equiv \sin \varphi=\sqrt{1-c^{2}},
$$


Near the logarithmic singularity, $m_{c}$ and $c$ are small while $s$ is close to unity.

The above approximations are effective when $m_{c} \tan ^{2} \varphi<1$, and therefore $c<$ $\sqrt{k_{c} /\left(1+k_{c}\right)}$ where $k_{c} \equiv \sqrt{m_{c}}$ is the complementary modulus. If $m_{c} \tan ^{2} \varphi>1$, on the other hand, the complementary forms become efficient. We shall not reproduce them here since they are easily derived from the special addition formulas such as the formula 116.01 of [2].

C.2. Expressions of Karp and Sitnik. Recently, Karp and his colleagues obtained the expressions similar to but slightly different from the classic expansions 29, 28. The main formulas for $F(\varphi \mid m)$ provided as equations (67) and (68) in [29] are rewritten as

$$
\begin{gathered}
F_{\mathrm{KS} 1}=\left(1+\frac{m_{c}}{4}\right) \log \left(\frac{1+s}{c}\right)+\frac{1}{s} \log \left(\frac{2 c}{c+d}\right), \\
F_{\mathrm{KS} 2}=\left(1+\frac{m_{c}}{4}+\frac{9 m_{c}^{2}}{64}\right) \log \left(\frac{1+s}{c}\right) \\
+\frac{1}{s}\left(1+\frac{m_{c}}{4}-\frac{c^{2}}{2 s^{2}}\right) \log \left(\frac{2 c}{c+d}\right)-\frac{m_{c} d}{4 s(c+d)},
\end{gathered}
$$

where

$$
d \equiv \sqrt{1-m \sin ^{2} \varphi}=\sqrt{m_{c}+m c^{2}},
$$

is another small quantity in the critical region. We do not quote their complements given as equations (69) and (71) in [29.

C.3. Expressions of Carlson and Gustafson. On the other hand, Carlson and Gustafson obtained different expressions in [13, 14]. They are rewritten in the case of $F(\varphi \mid m)$ as

$$
\begin{gathered}
F_{\mathrm{CG} 1}=s \log \left(\frac{4}{c+d}\right), \\
F_{\mathrm{CG} 2}=\left(1+\frac{w}{4}\right) s \log \left(\frac{4}{c+d}\right)+\frac{s(c d-w)}{4},
\end{gathered}
$$

where

$$
w \equiv c^{2}+d^{2},
$$

is a small quantity of the second order in the critical region. These expressions are effective in all the parameter domain. This uniformity is a noteworthy property of them.

C.4. New expressions. Our recipe in this case is the combination of the series expansions using $\left(u_{5}, v_{5}, a_{5}\right)$ and its complement $\left(u_{5 c}, v_{5 c}, a_{5 c}\right)$ depending on the ratio $x(y+z) /(y z)$ as explained in $\oint 3.7$. The former expansion of $R_{F}$ becomes

$$
R_{F}=I_{01}\left(u_{5}, v_{5}\right)-\left(\frac{a_{5}}{2}\right) I_{03}\left(u_{5}, v_{5}\right)+\left(\frac{3 a_{5}^{2}}{8}\right) I_{05}\left(u_{5}, v_{5}\right)+\cdots .
$$


Since $u_{5}>v_{5}$, the expression of $I_{01}\left(u_{5}, v_{5}\right)=R_{C}\left(u_{5}, v_{5}\right)$ reduces to that using the logarithm given in equation (A.5). Then the first three approximations of the corresponding series expansion of $F(\varphi \mid m)$ become

$$
\begin{gathered}
F_{\mathrm{F} 1}=\sqrt{\frac{2}{2-m_{c}}} \log \left(\frac{\sqrt{2}+\sqrt{2-w}}{\sqrt{w}}\right) \\
F_{\mathrm{F} 2}=\left(1+\frac{3 m_{c}^{2} s^{4}}{16(2-w)^{2}}\right) \sqrt{\frac{2}{2-m_{c}}} \log \left(\frac{\sqrt{2}+\sqrt{2-w}}{\sqrt{w}}\right) \\
+\frac{m_{c}^{2} s^{5}}{8 w(2-w)}\left(\frac{2}{w}-\frac{3}{2-w}\right) \\
F_{\mathrm{F} 3}=\left(1+\frac{3 m_{c}^{2} s^{4}}{16(2-w)^{2}}+\frac{105 m_{c}^{4} s^{8}}{1024(2-w)^{4}}\right) \sqrt{\frac{2}{2-m_{c}}} \log \left(\frac{\sqrt{2}+\sqrt{2-w}}{\sqrt{w}}\right) \\
+\frac{m_{c}^{2} s^{5}}{8 w(2-w)}\left(\frac{2}{w}-\frac{3}{2-w}\right) \\
+\frac{m_{c}^{4} s^{9}}{512 w(2-w)}\left(\frac{48}{w^{3}}-\frac{56}{w^{2}(2-w)}+\frac{70}{w(2-w)^{2}}-\frac{105}{(2-w)^{3}}\right)
\end{gathered}
$$

\section{ACKNOWLEDGMENTS}

We appreciate the reviewer's valuable suggestions including the subdivision of the splitting with the linear remainder of the first type into two cases that significantly improved the readability of this article.

\section{REFERENCES}

1. Abramowitz, M., \& Stegun, I.A. (eds.), Handbook of Mathematical Functions with Formulas, Graphs, and Mathematical Tables, Chapter 17. National Bureau of Standards, Washington (1964)

2. Byrd, P.F., \& Friedman, M.D., Handbook on Elliptic Integrals for Engineers and Physicists, 2nd ed. Springer-Verlag, Berlin (1971) MR0060642 (15:702a)

3. Bulirsch, R., Numerical Calculation of Elliptic Integrals and Elliptic Functions, Numer. Math., 7, 78-90 (1965a) MR0175284 (30:5469)

4. Bulirsch, R., Numerical Calculation of Elliptic Integrals and Elliptic Functions II, Numer. Math., 7, 353-354 (1965b) MR0185802 (32:3262)

5. Bulirsch, R., An Extension of the Bartky-Transformation to Incomplete Elliptic Integrals of the Third Kind, Numer. Math., 13, 266-284 (1969a) MR0247734(40:997)

6. Bulirsch, R., Numerical Calculation of Elliptic Integrals and Elliptic Functions III, Numer. Math., 13, 305-315 (1969b) MR0247735 (40:998)

7. Carlson, B.C., Normal Elliptic Integrals of the First and Second Kinds, Duke Math. J., 31, 405-419 (1964) MR0164067(29:1366)

8. Carlson, B.C., On Computing Elliptic Integrals and Functions, J. Math. and Phys., 44, 332345 (1965) MR0175285 (30:5470)

9. Carlson, B.C., Elliptic Integrals of the First Kind, SIAM J. Math. Anal., 8, 231-242 (1977) MR0430341 (55:3346)

10. Carlson, B.C., Short Proofs of Three Theorems on Elliptic Integrals, SIAM J. Math. Anal., 9, 524-528 (1978) MR0473247 (57:12922)

11. Carlson, B.C., Computing Elliptic Integrals by Duplication, Numer. Math., 33, 1-16 (1979) MR545738 (80h:65008) 
12. Carlson, B.C., \& Notis, E.M., Algorithm 577. Algorithms for Incomplete Elliptic Integrals, ACM Trans. Math. Software, 7, 398-403 (1981)

13. Carlson, B.C., \& Gustafson, J.L., Asymptotic Expansion of the First Elliptic Integral, SIAM J. Math. Anal., 16, 1072-1092 (1985) MR800798 (87d:33002)

14. Carlson, B.C., \& Gustafson, J.L., Asymptotic Approximations for Symmetric Elliptic Integrals, SIAM J. Math. Anal., 25, 288-303 (1994) MR1266560 (95b:33056)

15. Cody, W.J., Chebyshev Approximations for the Complete Elliptic Integrals K and E, Math. Comp., 19, 105-112 (1965a) MR0171370 (30:1601)

16. Cody, W.J., Chebyshev Polynomial Expansions of Complete Elliptic Integrals K and E, Math. Comp., 19, 249-259 (1965b) MR0178563(31:2820)

17. Cody, W.J., Corrigenda: Chebyshev Approximations for the Complete Elliptic Integrals K and E, Math. Comp., 20, 207 (1966) MR0171370 (30:1601)

18. Didonato, A.R., \& Hershey, A.V., New Formulas for Computing Incomplete Elliptic Integrals of the First and Second Kind, J. Assoc. Comput. Mach., 6, 515-526 (1959) MR0107353 (21:6078)

19. Fair, W.G., \& Luke, Y.L., Rational Approximations to the Incomplete Elliptic Integrals of the First and Second Kinds, Math. Comp., 21, 418-422 (1967) MR.0222348 (36:5400)

20. Ferreira, C., \& Lopez, J.L., Symmetric Standard Elliptic Integrals with Two or Three Large Parameters, Integral Transf. and Special Functions, 17, 433-442 (2006) MR2238580 (2007a:41051)

21. Frisch-Fay, R., Applications of Approximate Expressions for Complete Elliptic Integrals, Int. J. Mech. Sci., 5, 231-235 (1963)

22. Fukushima, T., Fast Computation of Complete Elliptic Integrals and Jacobian Elliptic Functions, Celest. Mech. Dyn. Astron., 105, 305-328 (2009) MR2559416 (2011b:33046)

23. Fukushima, T., Fast Computation of Incomplete Elliptic Integral of First Kind by Half Argument Transformation, Numer. Math., 116, 687-719 (2010a) MR2721638

24. Fukushima, T., Precise Computation of Acceleration due to Uniform Ring or Disk, Celest. Mech. Dyn. Astron., 108, 339-356 (2010b)

25. Fukushima, T., Precise and Fast Computation of General Complete Elliptic Integral of Second Kind, Math. Comp., in printing (2010c)

26. Fukushima, T., \& Ishizaki, H., Numerical Computation of Incomplete Elliptic Integrals of a General Form, Celest. Mech. Dyn. Astron., 59, 237-251 (1994) MR.1285916 (95c:65041)

27. Hofsommer, D.J., \& van de Riet, R.P., On the Numerical Calculation of Elliptic Integrals of the First and Second Kind and Elliptic Functions of Jacobi, Numer. Math., 5, 291-302 (1963) MR0166906 (29:4179)

28. Karp, D., Savenkova, A., \& Sitnik, S.M., Series Expansions for the Third Incomplete Elliptic Integral via Partial Fraction Decompositions, J. Comp. Appl. Math., 207, 331-337 (2007) MR2345251 (2009b:33030)

29. Karp, D., \& Sitnik, S.M., Asymptotic Approximations for the First Incomplete Elliptic Integral near Logarithmic Singularity, J. Comp. Appl. Math., 205, 186-206 (2007) MR2324834 (2008h:33045)

30. Lopez, J.L., Asymptotic Expansions of Symmetric Standard Elliptic Integrals, SIAM J. Math. Anal., 31, 754-775 (2000) MR1745143 (2000m:33025)

31. Lopez, J.L., Uniform Asymptotic Expansions of Symmetric Elliptic Integrals, Constructive Approx., 17, 535-559 (2001) MR 1845267 (2002e:33032)

32. Luke, Y.L., Approximations for Elliptic Integrals, Math. Comp., 22, 627-634 (1968) MR.0226825 (37:2412)

33. Luke, Y.L., Further Approximations for Elliptic Integrals, Math. Comp., 24, 191-198 (1970) MR0258243 (41:2890)

34. Morris, A.H. Jr., NSWC Library of Mathematics Subroutines, Tech. Rep. NSWCDD/TR92/425, 107-110. Naval Surface Warfare Center, Dahlgren (1993).

35. Olver, F.W.J., Lozier, D.W., Boisvert, R.F., \& Clark, C.W. (eds.), NIST Handbook of Mathematical Functions, Chapter 19. Cambridge Univ. Press, Cambridge (2010) MR2723248

36. Press, W.H., Flannery, B.P., Teukolsky, S.A., \& Vetterling, W.T., Numerical Recipes: the Art of Scientific Computing. Cambridge Univ. Press, Cambridge (1986) MR833288(87m:65001a)

37. Press W.H., Teukolsky S.A., Vetterling W.T., Flannery B.P., Numerical Recipes: the Art of Scientific Computing, 3rd ed.. Cambridge Univ. Press, Cambridge (2007) MR2371990 (2009b:65001) 
38. Van de Vel, H., On the Series Expansion Method for Computing Incomplete Elliptic Integrals of the First and Second Kinds, Math. Comp., 23, 61-69 (1969) MR.0239732 (39:1089)

39. Zill, D.G., \& Carlson, B.C., Symmetric Elliptic Integrals of the Third Kind, Math. Comp., 24, 199-214 (1970) MR0262553 (41:7159)

National Astronomical Observatory of Japan, 2-21-1, Ohsawa, Mitaka, Tokyo 1818588, JAPAN

E-mail address: Toshio.Fukushima@nao.ac.jp 